DOI: http://dx.doi.org/10.22198/rys.2018.71.a372

Artículos

\title{
Crecimiento, instituciones y grupos de poder. Los efectos olvidados en Sinaloa, 1994-2014
}

\author{
Growth, institutions and power groups. The forgotten \\ effects in Sinaloa, 1994-2014
}

\author{
Ezequiel Avilés Ochoa* \\ Gumaro Álvarez Vizcarra**
}

Resumen: en esta investigación se identifican, mediante un modelo no numérico, las relaciones de causalidad que revelan los olvidos de los grupos de poder al frente de las instituciones políticas y económicas en Sinaloa, y que explican la debilidad del crecimiento económico local. La contribución reside en el uso de una metodología que evalúa el desempeño económico a partir de la valoración subjetiva del pensamiento de los expertos, integrantes de la elite del poder. Para tal fin, se introducen postulados de la teoría de los subconjuntos borrosos y el modelo de efectos olvidados. Los resultados sugieren que las causas del rezago tecnológico son la frágil democracia y una participación ciudadana débil, que reducen el potencial de crecimiento al acumular

* Profesor-investigador de la Universidad de Occidente, unidad Culiacán. Boulevard Lola Beltrán s/n, edificio H, colonia 4 de Marzo, C. P. 80020. Culiacán, Sinaloa, México. Teléfono: (667) 759 1300, extensión 2302. Correo electrónico: ezequiel.aviles@udo.mx

** Profesor-investigador del Instituto Tecnológico y de Estudios Superiores de Monterrey, campus Sinaloa. Boulevard Pedro Infante 3773, edificio PEIS, C. P. 80100. Culiacán, Sinaloa, México. Teléfono: (667) 759 1600, extensión 1729. Correo electrónico: galvarez@itesm.mx

Autor para correspondencia: Ezequiel Avilés Ochoa. Correo electrónico: ezequiel.aviles@ udo.mx 
grados de olvido. La inversión deficiente en capital humano, en tecnología e investigación y desarrollo derivan en progreso técnico insuficiente, retraso educativo, estancamiento tecnológico y delincuencia.

Palabras clave: crecimiento económico; instituciones; grupos de poder; efectos olvidados; incidencia; subconjuntos borrosos.

Abstract: through a non-numerical model, this research identifies the relations of causality revealed by omissions of the power groups that are in charge of political and economic institutions in Sinaloa, and which account for the weak local economic growth. This contribution lies in the use of a methodology that evaluates economic performance based on the subjective assessment of experts' thinking, who are members of the power elite. For this purpose, postulates of the fuzzy subset theory and the forgotten effects model are introduced. The results suggest that the causes of technological backwardness are a fragile democracy and a weak citizen participation, which reduce growth potential by accumulating degrees of omission. Deficient investment in human capital, technology and research and development leads to insufficient technical progress, educational backwardness, technological stagnation and crime.

Key words: economic growth; institutions; power groups; forgotten effects; incidence; fuzzy subsets.

Recibido el 26 de enero de 2016. Aceptado el 7 de octubre de 2016.

\section{Introducción}

Las interrogantes principales en el campo del crecimiento económico y el progreso social son: ¿por qué crecen las economías?; ¿por qué 
algunas regiones son más pobres que otras?, y ¿cuáles son las políticas que permiten brindar mejores condiciones de vida? (Sala-i-Martin 2000, 9; Barro 1999, vii; Krugman 1999, 6; Acemoglu et al. 2005). Uno de los desafíos trascendentes de las ciencias sociales (Acemoglu 2009, 8-21) es esclarecer por qué algunas economías se expanden más rápido, en tanto que otras se estancan y son cada vez más pobres. Para responder a estas cuestiones y plantear alternativas de solución se utilizan modelos neoclásicos, que ilustran los mecanismos del crecimiento y la divergencia del ingreso, y también las causas que explican la escasez de convergencia.

En la literatura destacan los modelos de crecimiento exógeno, como los de Solow (1956) y Swan (1956), complementados por Cass (1965) y Koopmans (1965), quienes infieren que la producción se expande con la acumulación de capital físico y el trabajo. ${ }^{1}$ La productividad conjunta aumenta, a largo plazo, a una tasa dada de progreso técnico, la cual es exógena. El capital muestra rendimientos crecientes a escala; se alcanza una condición estacionaria, donde la relación capital-trabajo es constante, y el stock de capital crece en línea con el producto. El supuesto de rendimientos decrecientes o constantes a escala viabiliza la convergencia entre regiones, con diferencias en su dotación inicial de capital.

Romer (1986) y Lucas (1988) contrastan los modelos de crecimiento exógeno, y postulan que el crecimiento de largo plazo está determinado por factores endógenos. La acumulación de capital humano genera rendimientos constantes a escala, e incluso crecientes, en la función de producción. La presencia de externalidades en el stock de capital humano acumulado contribuye a mejorar el progreso técnico público, así como la inversión privada en conocimiento. La tasa de crecimiento a largo plazo es endógena; está en función de las decisiones de inversión en capital humano y en investigación y desarrollo (I\&D). Ello origina rendimientos constantes o crecientes, lo cual acelera el progreso técnico y la productividad total de los factores. En consecuencia, se manifiesta un proceso de divergencia, contrario a la hipótesis de los modelos exógenos. Los rendimientos crecientes, generados por las innovaciones técnicas, imposibilitan la convergencia de largo plazo.

1 Ros (2008) argumenta que el determinante principal de la desaceleración económica en México es una tasa baja de acumulación del capital físico. 
En fecha reciente, el debate incorporó el rol de las organizaciones en el crecimiento; se postula que las diferencias en las instituciones económicas y políticas son la causa principal de la divergencia o escasez de convergencia. Una lección de esta corriente es reconsiderar la importancia de las instituciones, ya que éstas inciden en la eficiencia de la economía, de manera similar a como lo hace la tecnología en el modelo de Solow y la acumulación de capital humano en el de Romer y Lucas.

North (1993) afirma que la teoría neoclásica es insuficiente para analizar y prescribir políticas que estimulen la dinámica productiva, porque está concentrada en explicar el funcionamiento de los mercados y no en cómo se expanden. Se arguye que los determinantes neoclásicos de crecimiento, tales como acumulación de capital, innovación, economías de escala, etcétera, más bien son manifestaciones del crecimiento (North y Thomas 1973, 2).

North (1994) formula un marco analítico donde la trasformación institucional es básica para explicar el progreso económico; precisa que los individuos edifican las instituciones para interactuar formalmente, y éstas definen los incentivos que, junto a otras restricciones, como el presupuesto y la tecnología, determinan las opciones de política diseñadas para estimular el progreso económico y social.

La discusión está centrada en examinar cómo la sociedad adopta y promueve políticas e instituciones ineficientes. Ello desincentiva la inversión en capital físico y humano, y se traduce en una dinámica productiva menor, salarios bajos y desocupación. Los grupos de poder dirigen las instituciones económicas y políticas y, por tanto, ejercen una influencia decisiva en la trayectoria del crecimiento y la distribución del ingreso; las últimas asignan el poder político de jure, mientras que las elites mantienen el de facto. Se afirma que la fragilidad de las democracias y el conflicto normal entre grupos de poder, sin compromiso explícito con el crecimiento de largo plazo, explican la incapacidad de las instituciones para promover un funcionamiento económico más robusto e incluyente (Acemoglu 2009, 831; Barro 1999, 61). Esta teoría traslada la discusión al interrogante de ipor qué ciertas instituciones políticas pueden mediar, con mayor éxito, el conflicto de luchas de poder y, por tanto, evitar la ejecución de políticas públicas que inhiban el crecimiento de largo plazo? 
El objetivo de la investigación es identificar, mediante un modelo no numérico, las relaciones de causalidad y los elementos interpuestos que evidencian los "olvidos" cometidos por los integrantes de la elite política al frente de las instituciones políticas y económicas, que explican el estancamiento productivo de la economía de Sinaloa en el contexto nacional y el noroeste de México.

La metodología contempla la condición subjetiva del pensamiento de los expertos, miembros de los grupos de poder que operan las instituciones. El conjunto de relaciones establecidas en su interacción se enmarca en un contexto mutable, donde la única constante es la entropía. Como elemento renovador se introducen los postulados de la lógica borrosa del modelo de efectos olvidados, desarrollado por Kaufmann y Gil Aluja (1988).

\section{Crecimiento económico, instituciones y grupos de poder: un enfoque heterodoxo}

Pumpler y Martin (2003) aseguran que desde Adam Smith se advierte la convicción de la influencia significativa de las instituciones económicas y políticas en el crecimiento. Se acepta que el progreso económico y social de una región depende significativamente del medio ambiente institucional y político ${ }^{2}$ en el que se despliega la actividad productiva; también de la participación ciudadana, que influye en la asignación del gasto público. Friedman (1962) estima que la calidad de la democracia, determinada por instituciones políticas eficaces, incide en el crecimiento, y arguye que la fortaleza democrática se manifiesta en la energía de las organizaciones económicas.

Swyngedouw (2000) apunta que la teoría institucional plantea que, además de las ventajas económicas, la estructura social, cultural e institucional explica la divergencia del crecimiento. La ordenación de

2 La expresión medio ambiente institucional y político hace referencia al conjunto de organizaciones públicas y privadas que influyen en el rumbo de la actividad económica, política y social de una región o país. North $(1990 ; 1981)$ las clasifica como un conjunto de reglas vigentes en la interacción social. Pumpler y Martin (2003), Friedman (1962) y Acemoglu (2009; et al. 2005) las conceptualizan como un conjunto de reglas, normas, procedimientos y conductas que promueven y rigen la interacción social. 
las instituciones determina la organización de las economías urbanas y regionales. La incrustación de los grupos de poder en las actividades productivas imposibilita la libre competencia obstaculizando la aparición de participantes nuevos. Las elites son capaces de acordar y crear una coalición coherente y estable para apropiarse de la trayectoria y beneficios del crecimiento, pese a sus posiciones heterogéneas y contradictorias.

North (1990) define a las instituciones como las reglas del juego vigentes en una sociedad; son las restricciones decretadas por los individuos para modelar su interacción. North también precisa que el marco institucional es un conjunto de reglas, procedimientos y normas de conducta, tanto éticas como morales, concebidas para restringir y organizar la conducta de los individuos, con el propósito de maximizar la producción de riqueza individual y la prosperidad social (1981).

Según Acemoglu (2009, 782), a las instituciones económicas les concierne la organización del sistema impositivo, el ejercicio presupuestal, la observancia del Estado de derecho y la salvaguarda de la competencia económica; a las instituciones políticas, les corresponde el ordenamiento y la reglamentación de las decisiones políticas y, al mismo tiempo, organizar, ordenar y agregar las opiniones diversas de los individuos y grupos sociales. Acemoglu et al. (2005) retoman el marco de referencia para examinar la influencia de los grupos de poder y de las instituciones en el crecimiento económico, que se puede resumir en que estas últimas alinean los incentivos otorgados a los actores clave de la sociedad; influyen en las inversiones en capital y tecnología física y humana y organizan la producción. Si bien los factores culturales y geográficos son importantes, las diferencias institucionales son fuente de la divergencia. Asimismo, determinan el potencial de crecimiento y la distribución del ingreso. Estas ideas se pueden expresar esquemáticamente como sigue:

$$
\text { Instituciones económicas }_{t} \rightarrow\left\{\begin{array}{l}
\text { crecimiento económico }_{t} \\
\text { distribución de recursos }_{t+1}
\end{array}\right.
$$

donde el subíndice $t$ se refiere al periodo $t+1$ actual para el futuro. 
Las instituciones económicas tienen un carácter endógeno; se fundan a partir de decisiones colectivas. Sin embargo, no hay garantía de que todos los individuos y grupos prefieran al mismo conjunto de ellas. En consecuencia, existe un conflicto en su conformación, y las de equilibrio están en función del poder político:

Poder político $_{\mathrm{t}} \longrightarrow$ instituciones económicas $_{\mathrm{t}}$

En la idea de que el poder político determina las instituciones económicas, está implícita la noción de la presencia de intereses en conflicto en la distribución de los recursos públicos. Los grupos que detentan el poder político lo utilizan para su interés superior, lo cual suscita disociación entre eficiencia y equidad.

Aunque el poder político se distribuye de manera endógena en la sociedad, es útil distinguir el de jure y el de facto; el primero se refiere a la energía originada en las instituciones políticas. De igual forma que las económicas determinan las limitaciones y los incentivos de los actores clave, pero esta vez en el ámbito político. Asimismo, circunscriben la forma de gobierno, por ejemplo, la democracia frente a la dictadura, y el alcance de las restricciones sobre los políticos y las elites. Por lo tanto, esta discusión implica que:

Instituciones políticas $_{\mathrm{t}} \rightarrow$ poder político de jure ${ }_{\mathrm{t}}$

El poder político reside formalmente en las instituciones políticas. Sin embargo, un grupo de individuos puede asumirlo, conservarlo y utilizarlo para imponer sus deseos sobre el resto de la sociedad. Este tipo de poder de facto depende de: a) la capacidad del grupo de resolver su problema de acción colectiva y b) la dotación de recursos económicos, que determina su capacidad de manejo de las instituciones políticas y de utilizar la fuerza contra los grupos opositores. Al no disponer de una teoría satisfactoria de cómo los grupos son capaces de resolver sus problemas de acción colectiva, la atención se centra en el poder de facto, por lo tanto:

Distribución de recursos $_{\mathrm{t}} \rightarrow$ poder político de facto $_{\mathrm{t}}$

En este sistema dinámico, las instituciones políticas y la distribución de los recursos son las variables clave, ya que determinan, de 
manera directa e indirecta, las instituciones económicas y la actividad productiva. Su efecto directo es que si colocan todo el poder en manos de una persona o grupo, las instituciones económicas no pueden garantizarle plenamente al resto de la población la protección de los derechos de propiedad y la igualdad de oportunidades. En cuanto al efecto indirecto, las instituciones políticas determinan la distribución del poder político de jure, que a su vez influye en la elección de las económicas. Por ende, se introduce un concepto natural de jerarquía de las políticas sobre las económicas, que luego determinan el desempeño económico de una región. Las instituciones políticas son endógenas, y se establecen a partir de decisiones colectivas. La distribución del poder político en la sociedad es el factor determinante del avance de las económicas. Las instituciones políticas asignan poder político de jure, y quienes tienen influencia en la evolución de éstas por lo general optan por mantenerlas. Sin embargo, de facto el poder político en ocasiones crea cambios en las instituciones políticas, lo que se resume así:

$$
\text { Poder político }_{\mathrm{t}} \rightarrow \text { instituciones políticas }_{\mathrm{t}+1}
$$

Figura 1. Representación esquemática del marco de referencia

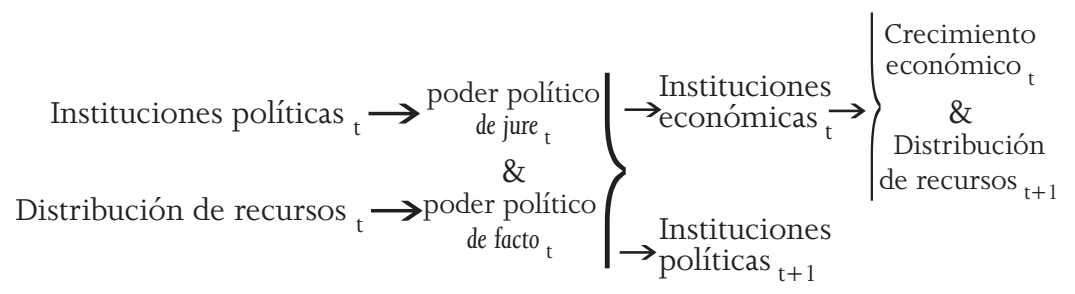

Fuente: elaboración con base en Acemoglu (2009; et al. 2005).

\section{El desempeño de la economía sinaloense, 1994-2014}

La economía de Sinaloa es una de las que tiene menor ritmo de expansión productiva de México. Desde la apertura comercial, en 1994, y hasta 2014, registró una tasa de crecimiento promedio de 2 por ciento, inferior a la del promedio nacional, de 2.7; se situó en el lugar 29 entre las entidades federativas (véase Figura 2). 


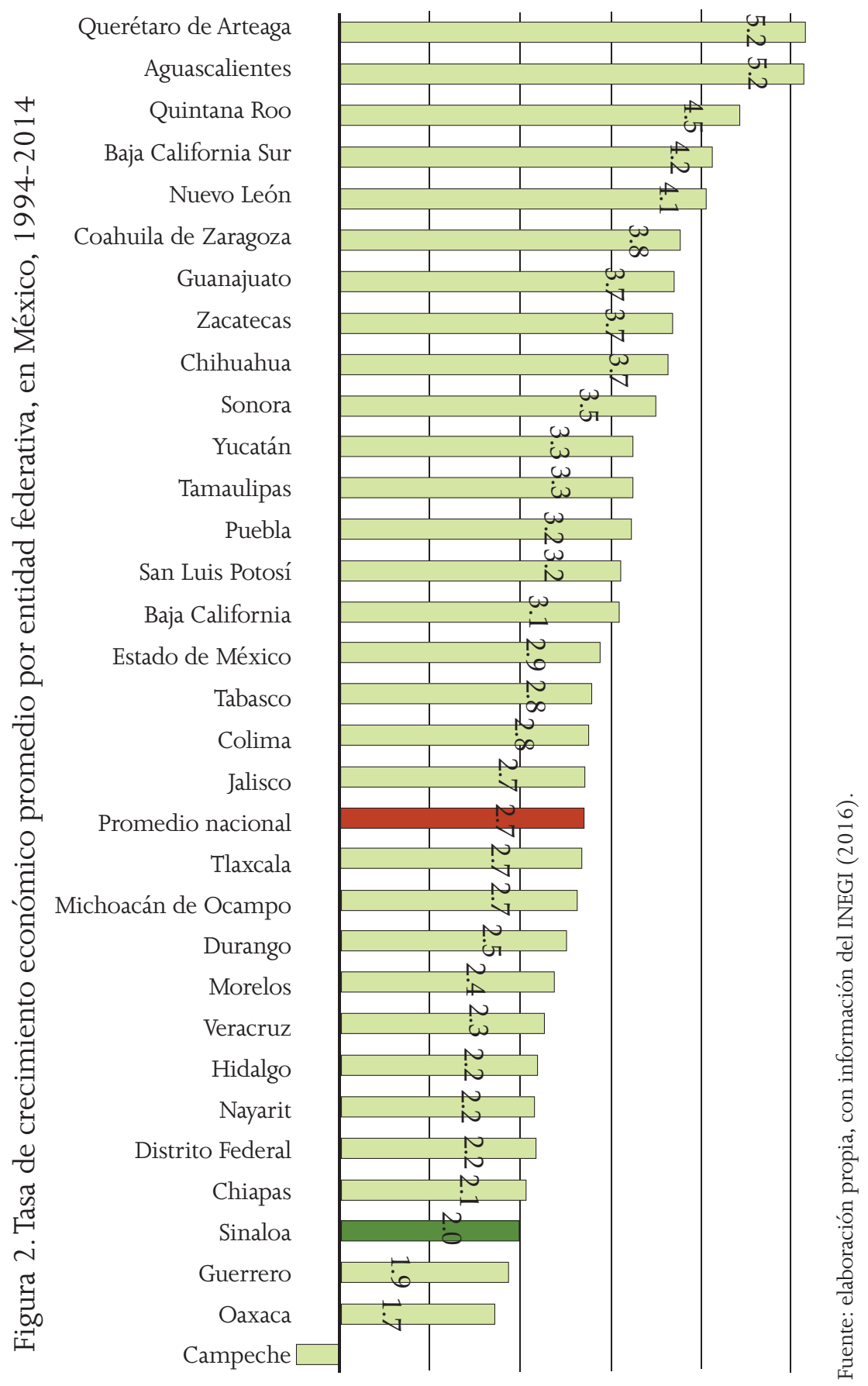


El crecimiento económico promedio de los estados del noroeste de México fue de 3.2 por ciento; el sinaloense fue el menos dinámico de la región (véase Figura 3).

Figura 3. Región noroeste de México. Crecimiento económico, 1994-2014 (promedio variación porcentual anual)

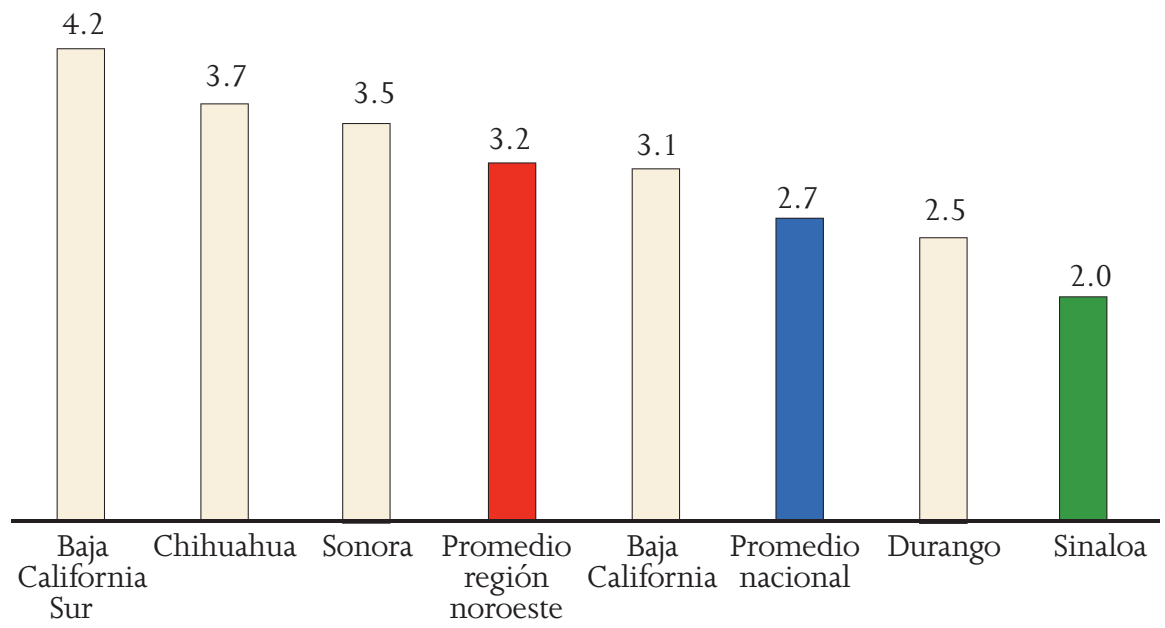

Fuente: elaboración propia, con información del INEGI (2016).

La tasa de crecimiento es muy volátil; además, en los últimos diez años registró una tendencia decreciente (véase Figura 4).

En el mediano y largo plazo, las diferencias en la velocidad del crecimiento del producto interno bruto por habitante (PIB per cápita) explican la divergencia en el nivel de vida entre regiones. El promedio nacional es 23 por ciento mayor al sinaloense. En el periodo 19932014, el nacional aumentó a un ritmo de 1.2 por ciento, mientras que el estatal lo hizo a uno de 0.8 ; la divergencia tiende a incrementarse en el tiempo (véase Figura 5). 


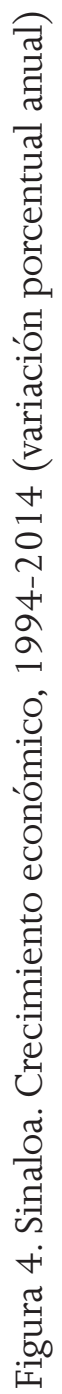

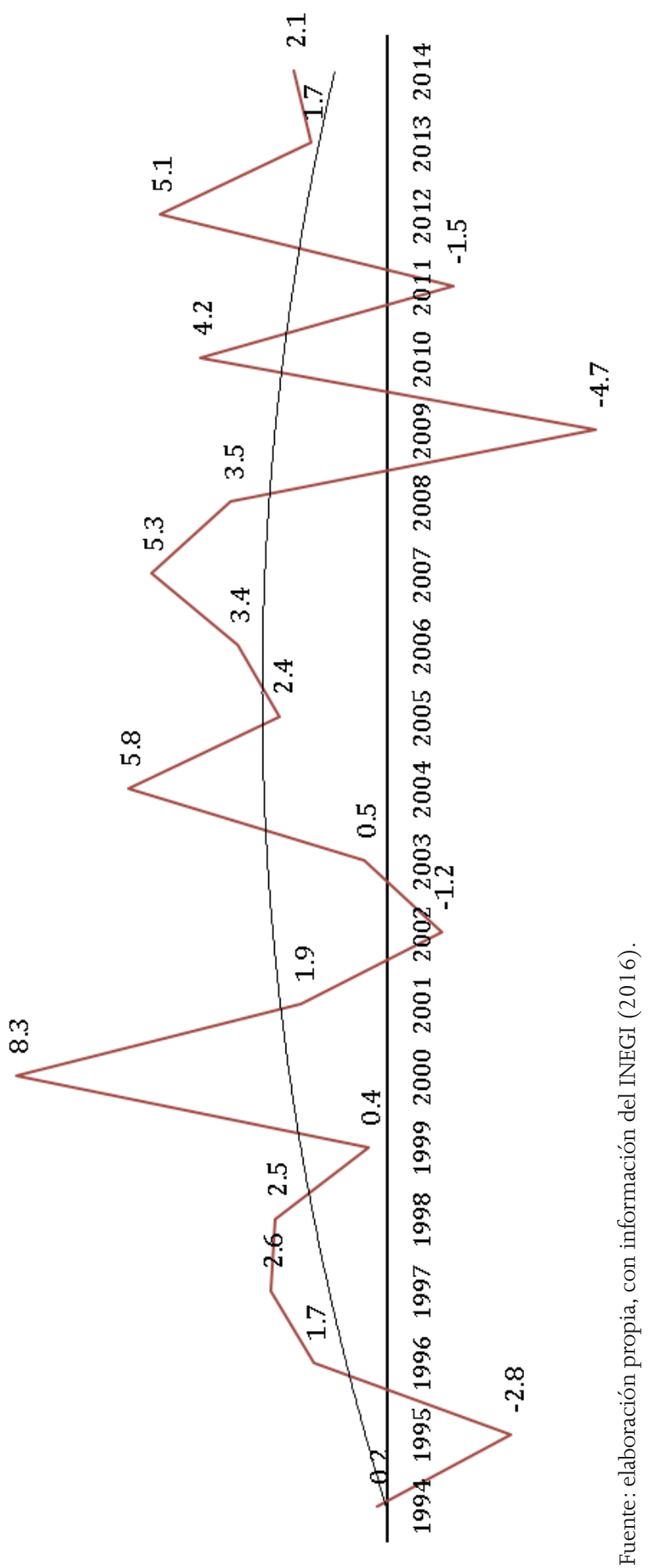


Figura 5. PIB per cápita nacional-Sinaloa, 1994-2038

(tendencia y proyecciones)

PIB per cápita nacional

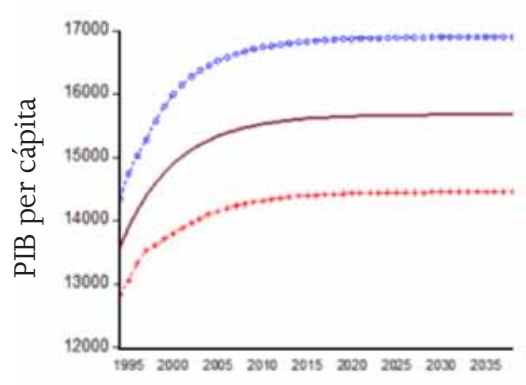

Año
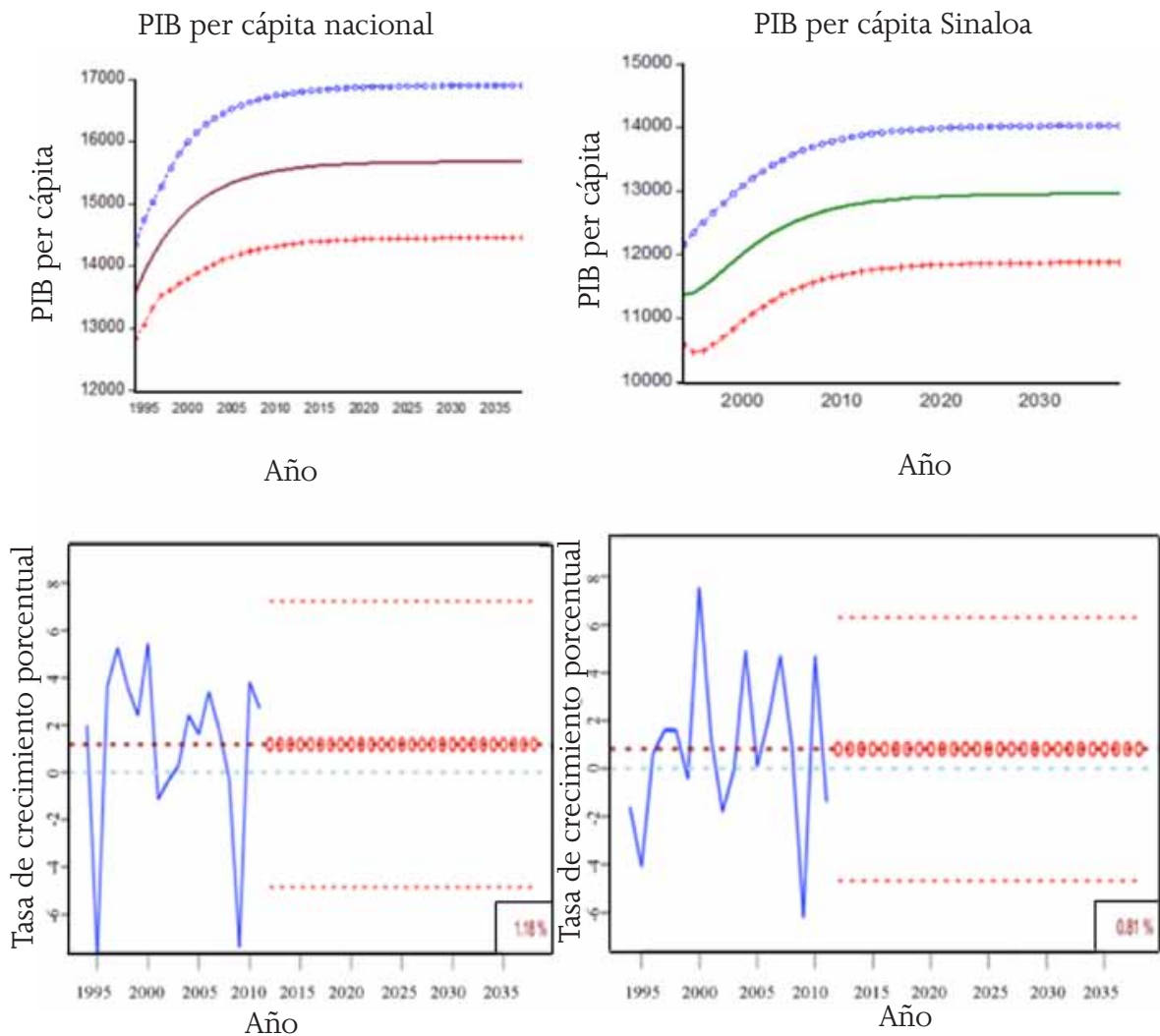

Fuente: elaboración propia, con información del INEGI (2016) y el Centro de Estudios de las Finanzas Públicas (CEFP 2016). El PIB per cápita está expresado en pesos de 1993.

\section{Estructura productiva}

La estructura productiva está concentrada en actividades con adición limitada de valor agregado y términos de intercambio desfavorables. Esta situación no se ha modificado significativamente durante la vigencia del Tratado de Libre Comercio de América del Norte. En Sinaloa, las actividades primarias continúan representando 21 por ciento del PIB, y 7.3 las de la industria manufacturera. También permanece estable la participación de 2.1 por ciento de la producción estatal en 
la nacional. La importancia del sector primario del estado es destacada, ya que ocupa el cuarto lugar en México; participa con 7.8 por ciento, más del doble del promedio del país, que es de 3.1. En la industria manufacturera ocurre lo opuesto, su contribución es de 1.2 por ciento, muy por debajo del promedio nacional, de 17 , esto la ubica en el lugar 22; la del sector servicios es de 2.2 por ciento, ocupa la posición 14; este ramo es el más dinámico, en especial el comercio, cuya participación en el PIB estatal es de 21 por ciento.

\section{Inversión extranjera directa}

Para los flujos de inversión privada nacional o extranjera no es atractiva una economía con predominio de actividades productivas con escasa incorporación de valor agregado, y sin perspectivas de cambio estructural en el mediano plazo. Una de las debilidades de la economía sinaloense reside en que su capacidad de atracción es limitada; tiene el menor flujo de inversión extranjera directa (IED) en el noroeste del país, representa sólo 3 por ciento (véase Figura 6).

Figura 6. Inversión extranjera directa de la región noroeste de México, 1994-2014 (participación)

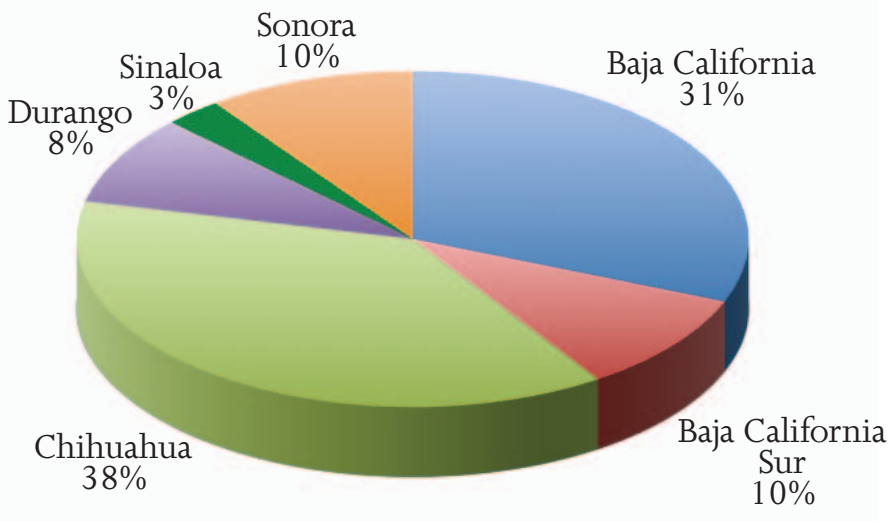

Fuente: elaboración propia, con información del INEGI (2016). 
De 1994 a 2014, la IED de Sinaloa registró un promedio anual de 73.4 millones de dólares; éste es el menor monto entre las entidades de la región (véase Figura 7).

Figura 7. Inversión extranjera directa promedio anual.

Región noroeste de México, 1994-2014 (millones de dólares)

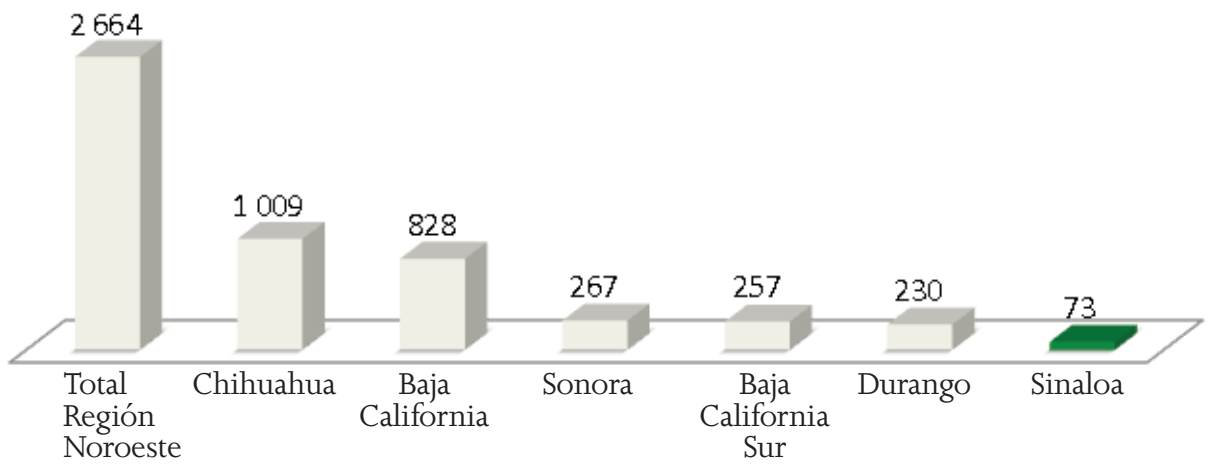

Fuente: elaboración propia, con información del INEGI (2016).

Si bien la participación porcentual promedio de la IED estatal en la nacional registró una tendencia creciente en los últimos años, sólo fue de 0.6 por ciento (véase Figura 8).

Figura 8. Inversión extranjera directa de Sinaloa, 1994-2014 (participación \%)

1.8

1.1

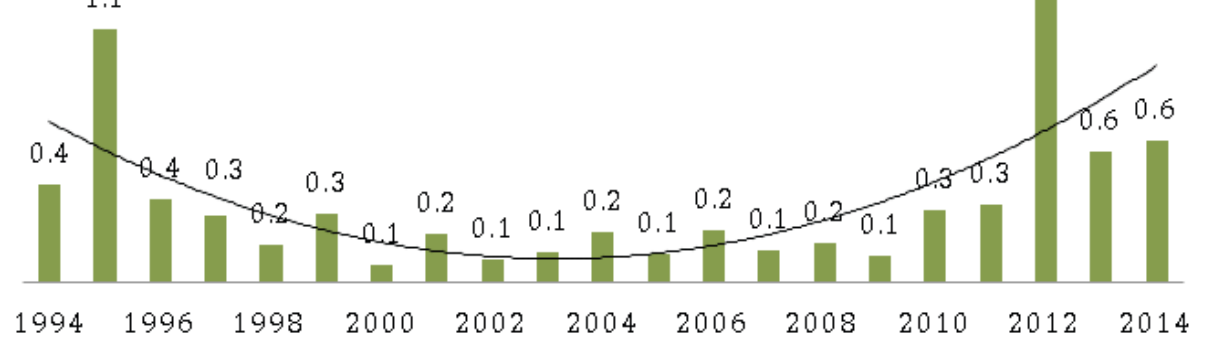

Fuente: elaboración propia, con información del INEGI (2016). 


\section{Pobreza y distribución del ingreso}

Según North y Thomas (1973), los grupos de poder que dirigen las instituciones económicas y políticas influyen significativamente en el crecimiento y la distribución del ingreso. Para Sinaloa, este postulado se cumple en el lapso en estudio, ya que el crecimiento insuficiente estuvo acompañado por una expansión de la pobreza y una desigualdad significativa del ingreso.

Figura 9. Evolución de la pobreza en Sinaloa, 2010-2014

\begin{tabular}{|c|c|c|c|c|c|c|}
\hline \multirow{2}{*}{ Indicadores } & \multicolumn{3}{|c|}{ Porcentaje } & \multicolumn{3}{|c|}{ Miles de personas } \\
\hline & 2010 & 2012 & 2014 & 2010 & 2012 & 2014 \\
\hline \multicolumn{7}{|c|}{ Pobreza } \\
\hline Población en situación de pobreza & 37 & 36 & 39 & 1049 & 1056 & 1167 \\
\hline $\begin{array}{c}\text { Población en situación } \\
\text { de pobreza moderada }\end{array}$ & 31 & 32 & 34 & 892 & 925 & 1011 \\
\hline $\begin{array}{c}\text { Población en situación } \\
\text { de pobreza extrema }\end{array}$ & 5 & 4 & 5 & 156 & 130 & 156 \\
\hline $\begin{array}{l}\text { Población vulnerable } \\
\text { por carencias sociales }\end{array}$ & 33 & 34 & 30 & 953 & 976 & 900 \\
\hline Población vulnerable por ingresos & 8 & 6 & 7 & 220 & 186 & 205 \\
\hline Población no pobre y no vulnerable & 22 & 24 & 23 & 638 & 693 & 693 \\
\hline \multicolumn{7}{|c|}{ Privación social } \\
\hline $\begin{array}{c}\text { Población con al menos } \\
\text { una carencia social }\end{array}$ & 70 & 70 & 70 & 2002 & 2032 & 2067 \\
\hline $\begin{array}{l}\text { Población con al menos } \\
\text { tres carencias sociales }\end{array}$ & 19 & 19 & 20 & 542 & 540 & 584 \\
\hline \multicolumn{7}{|c|}{ Indicadores de carencia social } \\
\hline Rezago educativo & 19 & 18 & 19 & 553 & 536 & 566 \\
\hline $\begin{array}{c}\text { Carencia por acceso a los servicios } \\
\text { de salud }\end{array}$ & 21 & 17 & 15 & 611 & 482 & 450 \\
\hline Carencia por acceso a la seguridad social & 54 & 53 & 49 & 1533 & 1534 & 1462 \\
\hline $\begin{array}{l}\text { Carencia por calidad y espacios } \\
\text { en la vivienda }\end{array}$ & 8 & 10 & 11 & 242 & 294 & 319 \\
\hline $\begin{array}{c}\text { Carencia por acceso a los servicios } \\
\text { básicos en la vivienda }\end{array}$ & 16 & 16 & 18 & 448 & 467 & 533 \\
\hline Carencia por acceso a la alimentación & 25 & 26 & 30 & 701 & 753 & 879 \\
\hline \multicolumn{7}{|c|}{ Bienestar } \\
\hline $\begin{array}{l}\text { Población con ingreso inferior } \\
\text { a la línea de bienestar mínimo }\end{array}$ & 14 & 13 & 13 & 405 & 390 & 399 \\
\hline $\begin{array}{c}\text { Población con ingreso inferior } \\
\text { a la línea de bienestar }\end{array}$ & 44 & 43 & 46 & 1268 & 1241 & 1372 \\
\hline
\end{tabular}

Fuente: elaboración propia, con información de CONEVAL (2014). 
De acuerdo con las estimaciones del Consejo de Evaluación de la Política Social (CONEVAL 20 I4), en Sinaloa, 39.4 por ciento de la población total -1 millón 167 personas- se encontraban en situación de pobreza, y 5.3 -155.8 mil sinaloenses- estaban en condiciones de pobreza extrema. Por lo general, la mayor parte vivía en la zona serrana (véase Figura 9). De acuerdo con los parámetros de medición, una persona se encuentra en situación de pobreza cuando tiene al menos una carencia social en educación, acceso a servicios de salud, seguridad social y alimentación, y también en calidad, espacios y servicios básicos en la vivienda. Asimismo, cuando su ingreso es insuficiente para adquirir los bienes y servicios que requiere para satisfacer sus necesidades alimentarias y no alimentarias.

El ingreso estatal estaba concentrado en los últimos dos deciles; 60 por ciento de los hogares sólo recibía 28 por ciento; la inequidad en la distribución de la riqueza era significativa (véase Figura 10).

Figura 10. Deciles de ingreso y coeficiente Gini en Sinaloa, 2010 y 2012 (precios constantes de 2012)

\begin{tabular}{|c|r|r|r|r|r|r|}
\hline \multirow{2}{*}{$\begin{array}{c}\text { Deciles de } \\
\text { hogares }\end{array}$} & \multicolumn{5}{|c|}{2010} & \multicolumn{3}{|c|}{2012} \\
\cline { 2 - 7 } & Hogares & \multicolumn{1}{|c|}{ Ingreso } & Promedio & Hogares & \multicolumn{1}{c|}{ Ingreso } & Promedio \\
\hline $\begin{array}{c}\text { Ingreso } \\
\text { corriente }\end{array}$ & 745678 & 28786378777 & 38604 & 788657 & 30282112584 & 38397 \\
\hline I & 74567 & 452137197 & 6064 & 78865 & 529786106 & 6718 \\
\hline II & 74567 & 860364991 & 11538 & 78865 & 907923143 & 11512 \\
\hline III & 74567 & 1164953497 & 15623 & 78865 & 1192171446 & 15117 \\
\hline IV & 74567 & 1469166619 & 19703 & 78865 & 1538023070 & 19502 \\
\hline V & 74567 & 1818622921 & 24389 & 78865 & 1925142658 & 24411 \\
\hline VI & 74567 & 2243858982 & 30092 & 78865 & 2359444746 & 29918 \\
\hline VII & 74567 & 2754027820 & 36934 & 78865 & 2946040756 & 37355 \\
\hline VIII & 74567 & 3433725338 & 46049 & 78865 & 3664270493 & 46463 \\
\hline IX & 74567 & 4671943840 & 62654 & 78865 & 4853335198 & 61540 \\
\hline X & 74575 & 9917577572 & 132988 & 78872 & 10365974968 & 131428 \\
\hline $\begin{array}{c}\text { Coeficiente } \\
\text { de Gini }\end{array}$ & & & 0.44 & & & 0.44 \\
\hline
\end{tabular}

Fuente: elaboración propia, con base en información del INEGI (2014). 


\section{La metodología: los efectos olvidados}

Gil Aluja (2005) señala que en la actualidad los esfuerzos se orientan a estructurar un marco teórico y metodológico que permita explicar, con mayor rigor analítico, los olvidos de los tomadores de decisiones en ambientes inciertos. Zadeh introdujo la teoría de los conjuntos borrosos, ${ }^{3}$ cuyo objetivo es proporcionar las bases del razonamiento aproximado utilizando premisas imprecisas como instrumento para formular conocimiento. Con el concepto de lógica borrosa se le da consistencia al pensamiento subjetivo; constituye una ampliación de la lógica clásica booleana y un complemento de la lógica discreta, que evoluciona del enfoque binario a las respuestas multivalentes. En el universo, los eventos se manifiestan según su grado de pertenencia; la borrosidad significa multivalencia, donde todo es cuestión de grado, incluso la verdad y la falsedad (Zadeh 1965; Kaufmann y Gil Aluja 1987; Gil Aluja 1999 y 1996; Barcellos 2010). La inclusión del pensamiento subjetivo en la toma de decisiones, a través del planteamiento multivalente de la lógica borrosa, entreabre la puerta para el análisis de las políticas públicas y la actuación de los grupos de poder, que determinan el crecimiento económico regional a largo plazo.

\section{Descripción del modelo}

En la actualidad, el entorno de los decisores es volátil, por lo que Gil Lafuente y Gil Lafuente (2007) advierten que el futuro inmediato es un mundo de incertidumbre e incógnitas. Kaufmann y Gil Aluja (1986) suponen que para lograr un mejor reflejo de la naturaleza contemporánea es conveniente utilizar la teoría de los subconjuntos borrosos. Cuando los decisores intentan examinar, explicar y resolver un problema, con frecuencia incurren en errores u omisiones. Kaufmann y Gil Aluja (1988) afirman que tales olvidos son naturales, ya que

3 El origen podría fijarse en 1922, cuando Lukasiewicz cuestionó la lógica clásica booleana (valores cierto y falso) y propuso una lógica de valores ciertos en el intervalo unidad, como generalización de su lógica trivaluada. En los años treinta se propusieron lógicas multivaluadas para un número indeterminado de valores ciertos (igual o mayor que 2), identificados por números racionales en el intervalo [0,1] (Zadeh 1965, 339). 
existe una interdependencia de causas y efectos. Los errores u omisiones revelados en una exposición aumentada no se detectan de inmediato, porque es habitual que estén ocultos, en virtud de que son efectos de una acumulación de causas. La inteligencia humana necesita apoyarse en herramientas y modelos capaces de crear una base técnica, sobre la cual pueda trabajar con la información disponible, contrastarla con la obtenida del entorno y apuntar las relaciones de causalidad directa e indirecta (Kaufmann y Gil Aluja 1988).

A partir de los problemas donde se plantean relaciones de impacto de un conjunto de expresiones sobre otro, o sobre sí mismo, surge el concepto de incidencia, que puede ligarse al de función, eminentemente subjetiva y difícil de cuantificar. Sin embargo, las incidencias se propagan de manera concatenada provocando que se obvien muchas etapas; se olvidan conclusiones, y así se originan los efectos de segunda generación, cuya idea relativa se debe a Fourastié, según lo señalan Kaufmann y Gil Aluja (1988). No obstante, la atención a las incidencias permite concebir la vida cotidiana, mejorar el desempeño razonado e influir en la toma de decisiones en todos los ámbitos de la gestión económica, financiera, política y gubernamental. La aplicación del modelo de efectos olvidados permite detectar los olvidos registrados en la relación causal del conjunto de eventos suscitados en el entorno.

De acuerdo con Kaufmann y Gil Aluja (1988), la expresión matemática del modelo se representa cuando el conjunto discreto de causas que evitan la implementación de políticas públicas efectivas es $C=\left\{c_{1}, c_{2}, c_{3, \ldots}, c_{n}\right\}$ que tienen una incidencia sobre otro conjunto de efectos identificados como resultado de la implementación de esas políticas públicas es $E=\left\{e_{1}, e_{2}, e_{3, \ldots}, e_{m}\right\}$. Se dice que $c_{1}$ tiene una incidencia sobre $e_{1}$ si el valor de la función característica de pertenencia del par $\left(c_{2}, c_{2}\right)$ y $\left(e_{2}, e_{2}\right)$ está valuado en el rango $[0,1]$, es decir, el grado de incidencia de cada $c_{i}$ sobre cada $e_{j}$ se expresa a través de una función:

$$
\mu: C \rightarrow E \in[0,1]
$$

donde $\mu$ representa los valores de plano cartesiano formado por el conjunto $C$ y E determinados en el rango $[0,1]$ (Kaufmann y Gil Aluja 1988; Gil Lafuente y Bassa 2001). 
De ese modo:

$$
\forall\left(c_{i,} c_{j}\right) \in C \rightarrow E, \mu\left(c_{i}, e_{j}\right) \in[0,1]
$$

En forma ordinaria, $c_{n} \in C$ tiene una influencia sobre $e_{m} \in E$ si el valor par $\left(c_{n}, e_{m}\right)=1$; en el caso de no incidencia, el valor será $=0$. Cuando se le solicita al experto valuar ${ }^{4}$ la incidencia de $C$ sobre $E$, en valores binarios como ( 0 ó 1 ) encontrará cierta dificultad al expresar su apreciación, pues no siempre localizará la relación tan definida. Por tanto, se demuestra la conveniencia de emplear las matrices borrosas, con los valores del rango $[0,1]$ que le permiten al experto tener 11 opciones para evaluar la incidencia entre $c_{n}$ y $e_{m}$. El conjunto de pares valuados de esta manera conforman lo que se llama matriz de incidencia original, la cual se denota como [CE].

Para conocer los efectos de segundo orden se utilizan al menos tres matrices: $[C E],[C C],[E E]$, aplican un proceso de convolución matricial con el modelo maxmin, y se ponen en práctica dos operadores: (v) que significa máximo, el más grande de los números que se consideran, y $(\Lambda)$ el mínimo. Una vez que se tienen las notaciones de cada casilla, proporcionadas por el grupo de expertos, se realiza el procedimiento maxmin para conocer el valor de cada casilla de la matriz $[M *]=[C E] \circ[C C] \circ[E E]$ (donde ${ }^{\circ}$ denota la aplicación de la composición maxmin) La ecuación a partir de la cual se estiman los valores de $\left[M^{*}\right]$, se expresa como sigue:

$$
\begin{aligned}
\mu\left(c_{1}, b_{1}\right) & =\left(\mu\left(c_{1}, e_{1}\right) \wedge \mu\left(c_{1}, c_{1}\right)\right) \vee\left(\mu\left(c_{1}, e_{2}\right) \wedge \mu\left(c_{1}, e_{2}\right)\right) \vee\left(\mu\left(c_{1}, e_{3}\right) \wedge \mu\left(c_{1}, c_{3}\right)\right) \vee\left(\mu\left(c_{1}, e_{4}\right) \wedge \mu\left(c_{1}, c_{4}\right)\right) \\
= & (0 \wedge 0) \vee(1 \wedge 0) \vee(0 \wedge 1) \vee(0 \wedge 1) \\
= & (0) \vee(0) \vee(0) \vee(0)=0 \\
& \mu\left(c_{1}, b_{1}\right)=0
\end{aligned}
$$

4 El concepto valuación se asimila a una asignación numérica realizada subjetivamente. La subjetividad es la característica que la diferencia de la noción de medida, de carácter objetivo (Gil Aluja et al. 2004). 
La fórmula general del procedimiento se define así:

$$
\mu\left(c_{n}, b_{k}\right)=\mathrm{v}_{m}\left(\mu\left(c_{n}, e_{m}\right) \wedge \mu\left(e_{m}, b_{k}\right)\right)
$$

Se trabaja de igual forma, sin importar si son matrices ordinarias o borrosas. Las relaciones entre los conjuntos de variables en una matriz borrosa y un grafo sagitado se expresan así:

$$
\forall\left(c_{n}, b_{k}\right) \in M: v\left(c_{n}, b_{k}\right) \in[0,1]
$$

Kaufmann y Gil Aluja (1988) postulan que cuando a los expertos se les pide evaluar relaciones entre entidades de dos conjuntos, por medio de valores entre [o y 1], su consideración se encuentra matizada, lo cual posibilita localizar grados de verdad en la noción de incidencia. Se utiliza una escala semántica endecadaria, propuesta por Kaufmann y Gil Aluja (1988), que proporciona dos ventajas: a) permite, mediante la trasformación semántica, modificar fielmente las opiniones de los encuestados en valores numéricos y b) como está constituida por 11 valores $[0,0.1,0.2,0.3,0.4, \ldots 1]$, admite una mejor adaptación y tratamiento de los conceptos analizados, ya que las personas están acostumbradas a pensar y trabajar en forma decimal (Gil Lafuente y Bassa 2011).

A partir de la construcción de la matriz de efectos secundarios, es posible percibir los efectos olvidados. Para ello es necesario separar los efectos acumulados que aparecen en la matriz $\left[M^{*}\right]$, de los directos en la matriz $[C E]$. Esto es posible al realizar una resta algebraica simple: $\left[M^{* *}\right]=\left[M^{*}\right]-[C E]$. En síntesis, el modelo define un proceso de composición, donde a partir de tres matrices originales se generan dos más, denominadas: efectos secundarios y efectos olvidados. La composición se realiza al seleccionar la columna de una matriz A y la fila de una matriz B, luego, se comparan los valores de cada celda, se selecciona el valor mínimo de cada par, y después se elige el máximo de los mínimos, que representará el valor de una celda y de la matriz nueva. El proceso se repite hasta completar la nueva matriz.

En materia de políticas públicas, la aplicación del modelo de efectos olvidados, para detectar las omisiones de las instituciones y grupos de poder, permite advertir el origen de los eventos que obstaculizan el crecimiento económico. 


\section{Decisiones metodológicas}

El quehacer científico demanda formas menos ortodoxas, donde lo cuantitativo pueda complementarse con lo cualitativo. En este sentido, Kaufmann y Gil Aluja (1990) postulan que los enfoques nuevos deben estar orientados por las propiedades definidas en la teoría de los subconjuntos borrosos, cuya utilización permite aglutinar la subjetividad como una cuasi verdad. El trabajo de campo se realiza según el esquema expuesto en la Figura 11.

Figura 11. Seguimiento del proceso de investigación de campo

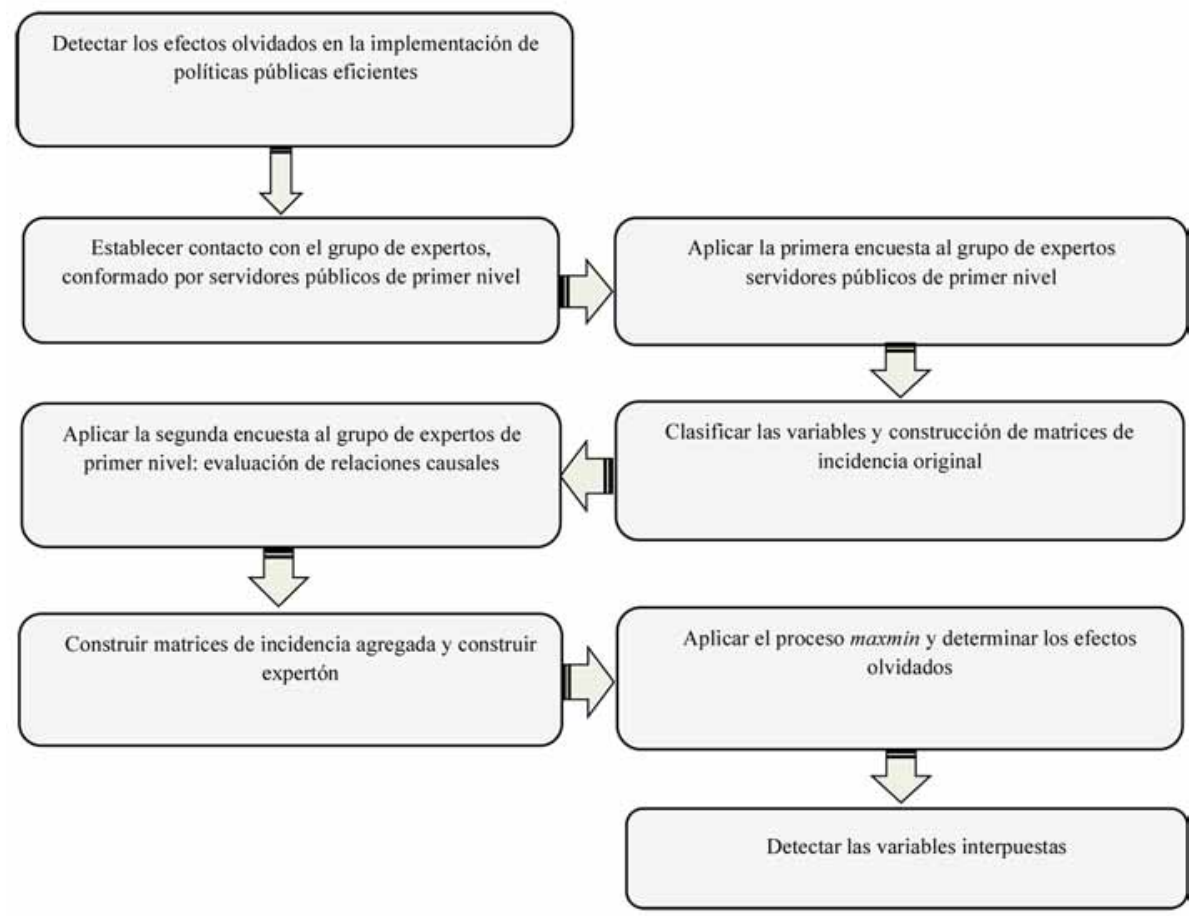

Fuente: elaboración propia. 


\section{Conformación del grupo de expertos} (los integrantes de la elite del poder)

La implementación del modelo de los efectos olvidados requiere trabajar con grupos de expertos. Kaufmann y Gil Aluja (1993) afirman que el punto de partida en todo proceso de búsqueda de conocimiento nuevo, destrezas y habilidades sobre un fenómeno es la comparación, a través del conocimiento abstracto o concreto. Por lo general, se recurre a especialistas cuando se trata de tomar ciertas decisiones importantes, lo que obliga a hacer comparaciones y agregaciones.

Para conformar al grupo se utiliza un muestreo no probabilístico del tipo de informante clave, consistente en seleccionar a personas vinculadas con grupos de poder político-económico y participación activa acreditada en la toma de decisiones. La selección se realizó de manera determinística convencional. A partir de las ideas de Ericsson et al. (2007), Tichy (2004) y Salazar (2012), un experto o miembro de la elite del poder es quien reúna alguna o varias de las características siguientes: a) estar activo en el servicio público; b) ocupar algún puesto de elección popular o haberlo tenido; c) pertenecer a una institución pública, privada, educativa o social; d) contar con experiencia en el área de gobierno y e) ser considerado líder de opinión.

Fueron 50 las personas que conformaron el grupo de expertos de la elite del poder; estuvo caracterizado por la pluralidad de opiniones, que evitó el posible sesgo generado por apegos políticos (véase Figura 12).

Figura 12. Miembros de la elite del poder en Sinaloa considerados en la muestra

\begin{tabular}{|c|c|c|}
\hline Experto & Instituciones y grupos & Porcentaje \\
\hline 1 & Exgobernadores & 4 \\
\hline 2 & Secretarios del gobierno estatal & 18 \\
\hline 3 & Presidentes y expresidentes municipales & 22 \\
\hline 4 & Legisladores y exlegisladores locales & 8 \\
\hline 5 & Dirigentes políticos & 24 \\
\hline 6 & Dirigentes universitarios & 14 \\
\hline 7 & Organismos sociales y colegios de profesionistas & 10 \\
\hline
\end{tabular}

Nota: las entrevistas se realizaron en mayo y junio de 2015.

Fuente: elaboración propia. 


\section{Definición de variables}

A partir de la literatura se precisó un conjunto de variables consideradas como causas, y otras como efectos de presencia o ausencia de crecimiento económico. En adelante se utiliza la notación $c_{i}$ para las causas $i=1,2,3, \ldots, n$, y $e_{j}$ para los efectos $j=1,2,3, \ldots, m$. A partir de estos hallazgos se construyeron las matrices de incidencia directa, cuyo eje vertical fueron las causas y el horizontal los efectos $\left(C^{*} E\right)$ (véase Figura 13).

Figura 13. Variables valuadas por los expertos

(integrantes de la elite del poder en Sinaloa)

\begin{tabular}{|c|c|c|c|}
\hline & Causas & & Efectos \\
\hline$C_{1}$ & Instituciones económicas & $E_{1}$ & Riqueza individual \\
\hline$C_{2}$ & Instituciones políticas & $E_{2}$ & Prosperidad social \\
\hline$C_{3}$ & Incentivos al trabajo & $E_{3}$ & Organización productiva \\
\hline$C_{4}$ & Presupuesto público & $E_{4}$ & Desigualdad regional \\
\hline$C_{5}$ & Tecnología & $E_{5}$ & Concentración de la riqueza \\
\hline$C_{6}$ & Grupos de poder & $E_{6}$ & Incremento de la delincuencia \\
\hline$C_{7}$ & Baja inversión en capital físico & $E_{7}$ & Pobreza \\
\hline$C_{8}$ & Baja inversión & $E_{8}$ & Retraso educativo \\
\hline$C_{9}$ & Frágil democracia & $E_{9}$ & Estancamiento tecnológico \\
\hline$C_{10}$ & Conflicto entre grupos & $E_{10}$ & Inestabilidad social \\
\hline$C_{11}$ & de poder & $E_{11}$ & Problemas laborales \\
\hline$C_{12}$ & Factores culturales & $E_{12}$ & Disminución de la inversión \\
\hline$C_{13}$ & Factores geográficos & $E_{13}$ & Progreso técnico \\
\hline$C_{14}$ & $\begin{array}{c}\text { Inversión en investigación } \\
\text { y desarrollo }\end{array}$ & $E_{14}$ & Incremento en la productividad \\
\hline & $E_{15}$ & Desempleo \\
\hline
\end{tabular}

Fuente: elaboración propia. 


\section{Construcción de matrices de incidencia original}

Como resultado de la aplicación de la segunda encuesta, como se describió en la Figura 11, se obtuvieron las valuaciones emitidas por los expertos con la escala endecadaria, en la cual se establecieron valores en el rango [0 1 1]. Estas valuaciones adquieren credibilidad por la diversidad y conocimiento de los expertos, la participación de encuestadores con experiencia en investigación cualitativa y por el resultado del modelo de agregación.

A partir de lo anterior se construyen las matrices agregadas $\left[C E^{\mathrm{A}}\right]$, $\left[C C^{\mathrm{A}}\right],\left[E E^{\mathrm{A}}\right]$. empleando un agregador de promedio simple, y validando el resultado por medio de un expertón (véase Figura 14, 15 y 16).

Figura 14. Incidencia entre causas y efectos

\begin{tabular}{|c|c|c|c|c|c|c|c|c|c|c|c|c|c|c|c|}
\hline & $E_{1}$ & $E_{2}$ & $E_{3}$ & $E_{4}$ & $E_{5}$ & $E_{6}$ & $E_{7}$ & $E_{8}$ & $E_{9}$ & $E_{10}$ & $E_{11}$ & $E_{12}$ & $E_{13}$ & $E_{14}$ & $E_{15}$ \\
\hline$C_{1}$ & 0,5 & 0,7 & 0,7 & 0,6 & 0,6 & 0,4 & 0,5 & 0,5 & 0,5 & 0,5 & 0,5 & 0,6 & 0,5 & 0,7 & 0,6 \\
\hline$C_{2}$ & 0,5 & 0,6 & 0,5 & 0,6 & 0,6 & 0,5 & 0,6 & 0,6 & 0,5 & 0,7 & 0,5 & 0,5 & 0,4 & 0,5 & 0,5 \\
\hline$C_{3}$ & 0,6 & 0,7 & 0,6 & 0,4 & 0,4 & 0,4 & 0,5 & 0,4 & 0,4 & 0,4 & 0,6 & 0,4 & 0,5 & 0,8 & 0,5 \\
\hline$C_{4}$ & 0,5 & 0,7 & 0,6 & 0,7 & 0,5 & 0,5 & 0,6 & 0,6 & 0,6 & 0,6 & 0,4 & 0,5 & 0,6 & 0,6 & 0,6 \\
\hline$C_{5}$ & 0,6 & 0,6 & 0,7 & 0,5 & 0,5 & 0,3 & 0,4 & 0,6 & 0,6 & 0,3 & 0,3 & 0,4 & 0,8 & 0,8 & 0,5 \\
\hline$C_{6}$ & 0,7 & 0,5 & 0,5 & 0,6 & 0,7 & 0,6 & 0,6 & 0,6 & 0,5 & 0,7 & 0,5 & 0,6 & 0,4 & 0,4 & 0,5 \\
\hline$C_{7}$ & 0,4 & 0,5 & 0,4 & 0,6 & 0,5 & 0,6 & 0,7 & 0,6 & 0,7 & 0,6 & 0,6 & 0,7 & 0,5 & 0,6 & 0,7 \\
\hline$C_{8}$ & 0,6 & 0,5 & 0,5 & 0,7 & 0,6 & 0,7 & 0,7 & 0,8 & 0,6 & 0,6 & 0,7 & 0,7 & 0,5 & 0,6 & 0,8 \\
\hline$C_{9}$ & 0,5 & 0,6 & 0,5 & 0,7 & 0,7 & 0,6 & 0,6 & 0,6 & 0,5 & 0,7 & 0,5 & 0,6 & 0,4 & 0,4 & 0,5 \\
\hline$C_{10}$ & 0,5 & 0,5 & 0,5 & 0,6 & 0,6 & 0,6 & 0,6 & 0,5 & 0,4 & 0,7 & 0,5 & 0,6 & 0,4 & 0,5 & 0,6 \\
\hline$C_{11}$ & 0,4 & 0,8 & 0,6 & 0,5 & 0,4 & 0,4 & 0,5 & 0,5 & 0,4 & 0,6 & 0,4 & 0,4 & 0,4 & 0,5 & 0,4 \\
\hline$C_{12}$ & 0,5 & 0,6 & 0,6 & 0,5 & 0,5 & 0,5 & 0,5 & 0,5 & 0,5 & 0,5 & 0,4 & 0,4 & 0,4 & 0,5 & 0,4 \\
\hline$C_{13}$ & 0,5 & 0,5 & 0,5 & 0,6 & 0,6 & 0,5 & 0,5 & 0,5 & 0,5 & 0,5 & 0,4 & 0,5 & 0,4 & 0,4 & 0,5 \\
\hline$C_{14}$ & 0,6 & 0,7 & 0,7 & 0,5 & 0,5 & 0,3 & 0,5 & 0,6 & 0,7 & 0,5 & 0,4 & 0,6 & 0,7 & 0,7 & 0,5 \\
\hline
\end{tabular}

Fuente: elaboración propia, a partir de las valuaciones vertidas por los expertos. Se utiliza la notación de coma en lugar de punto, porque el programa FuzzyLog ${ }^{5}$ así lo requiere.

5 Es un programa de cálculo que permite elaborar y trabajar con modelos basados en matemática de la incertidumbre, que agiliza el procesamiento de datos, reduce el margen de error y garantiza el acceso a resultados confiables para recuperar los efectos olvidados en las relaciones de causalidad. Los derechos de uso corresponden a la doctora Ana María Gil Lafuente. http:// www.fuzzyeconomics.com/fuzzylog 
Figura 15. Incidencias entre las causas

\begin{tabular}{|l|r|r|r|r|r|r|r|r|r|r|r|r|r|r|}
\hline $\boldsymbol{I}$ & $\boldsymbol{C}_{\boldsymbol{1}}$ & $\boldsymbol{C}_{\boldsymbol{2}}$ & $\boldsymbol{C}_{\boldsymbol{3}}$ & $\boldsymbol{C}_{\boldsymbol{4}}$ & $\boldsymbol{C}_{\boldsymbol{5}}$ & $\boldsymbol{C}_{\boldsymbol{6}}$ & $\boldsymbol{C}_{\boldsymbol{7}}$ & $\boldsymbol{C}_{\boldsymbol{8}}$ & $\boldsymbol{C}_{\boldsymbol{9}}$ & $\boldsymbol{C}_{\boldsymbol{1 0}}$ & $\boldsymbol{C}_{\boldsymbol{1 1}}$ & $\boldsymbol{C}_{\boldsymbol{1 2}}$ & $\boldsymbol{C}_{\boldsymbol{1 3}}$ & $\boldsymbol{C}_{\boldsymbol{1 4}}$ \\
\hline $\boldsymbol{C}_{\boldsymbol{1}}$ & 1 & 0,7 & 0,6 & 0,6 & 0,6 & 0,6 & 0,6 & 0,6 & 0,5 & 0,5 & 0,5 & 0,5 & 0,4 & 0,6 \\
\hline $\boldsymbol{C}_{\boldsymbol{2}}$ & 0,7 & 1 & 0,5 & 0,8 & 0,4 & 0,7 & 0,5 & 0,5 & 0,7 & 0,7 & 0,7 & 0,6 & 0,4 & 0,5 \\
\hline $\boldsymbol{C}_{\boldsymbol{3}}$ & 0,6 & 0,4 & 1 & 0,4 & 0,5 & 0,4 & 0,5 & 0,6 & 0,4 & 0,4 & 0,4 & 0,4 & 0,4 & 0,5 \\
\hline $\boldsymbol{C}_{\boldsymbol{4}}$ & 0,6 & 0,7 & 0,6 & 1 & 0,7 & 0,6 & 0,6 & 0,6 & 0,6 & 0,6 & 0,5 & 0,5 & 0,4 & 0,6 \\
\hline $\boldsymbol{C}_{\boldsymbol{5}}$ & 0,6 & 0,4 & 0,5 & 0,5 & 1 & 0,3 & 0,5 & 0,6 & 0,4 & 0,3 & 0,4 & 0,5 & 0,4 & 0,7 \\
\hline $\boldsymbol{C}_{\boldsymbol{6}}$ & 0,6 & 0,7 & 0,4 & 0,5 & 0,4 & 1 & 0,4 & 0,4 & 0,6 & 0,8 & 0,5 & 0,4 & 0,3 & 0,4 \\
\hline $\boldsymbol{C}_{\boldsymbol{7}}$ & 0,6 & 0,4 & 0,5 & 0,5 & 0,5 & 0,3 & 1 & 0,5 & 0,4 & 0,4 & 0,3 & 0,4 & 0,4 & 0,6 \\
\hline $\boldsymbol{C}_{\boldsymbol{8}}$ & 0,5 & 0,4 & 0,6 & 0,5 & 0,5 & 0,4 & 0,5 & 1 & 0,5 & 0,4 & 0,5 & 0,5 & 0,4 & 0,6 \\
\hline $\boldsymbol{C}_{\boldsymbol{9}}$ & 0,5 & 0,7 & 0,4 & 0,5 & 0,4 & 0,7 & 0,4 & 0,4 & 1 & 0,7 & 0,7 & 0,5 & 0,4 & 0,4 \\
\hline $\boldsymbol{C}_{\boldsymbol{1 0}}$ & 0,5 & 0,7 & 0,4 & 0,5 & 0,3 & 0,8 & 0,4 & 0,4 & 0,7 & 1 & 0,6 & 0,5 & 0,4 & 0,4 \\
\hline $\boldsymbol{C}_{11}$ & 0,5 & 0,7 & 0,5 & 0,6 & 0,4 & 0,5 & 0,4 & 0,4 & 0,7 & 0,6 & 1 & 0,6 & 0,4 & 0,5 \\
\hline $\boldsymbol{C}_{\boldsymbol{1 2}}$ & 0,5 & 0,5 & 0,4 & 0,5 & 0,5 & 0,5 & 0,5 & 0,5 & 0,6 & 0,5 & 0,6 & 1 & 0,5 & 0,5 \\
\hline $\boldsymbol{C}_{\boldsymbol{1 3}}$ & 0,4 & 0,5 & 0,4 & 0,5 & 0,5 & 0,4 & 0,5 & 0,5 & 0,4 & 0,4 & 0,4 & 0,5 & 1 & 0,4 \\
\hline $\boldsymbol{C}_{\boldsymbol{1 4}}$ & 0,6 & 0,4 & 0,5 & 0,5 & 0,8 & 0,4 & 0,6 & 0,6 & 0,4 & 0,4 & 0,4 & 0,5 & 0,5 & 1 \\
\hline
\end{tabular}

Fuente: elaboración propia, a partir de las valuaciones vertidas por los expertos.

Figura 16. Incidencias entre los efectos

\begin{tabular}{|c|c|c|c|c|c|c|c|c|c|c|c|c|c|c|c|}
\hline & $E_{1}$ & $E_{2}$ & $E_{3}$ & $E_{4}$ & $E_{5}$ & $E_{6}$ & $E_{7}$ & $E_{8}$ & $E_{q}$ & $E_{10}$ & $E_{11}$ & $E_{12}$ & $E_{13}$ & $E_{14}$ & $E_{15}$ \\
\hline$E_{1}$ & 1 & 0,6 & 0,6 & 0,6 & 0,8 & 0,6 & 0,6 & 0,5 & 0,4 & 0,5 & 0,5 & 0,5 & 0,5 & 0,6 & 0,5 \\
\hline$E_{2}$ & 0,5 & 1 & 0,6 & 0,6 & 0,6 & 0,5 & 0,5 & 0,5 & 0,5 & 0,5 & 0,4 & 0,4 & 0,6 & 0,7 & 0,5 \\
\hline$E_{3}$ & 0,5 & 0,6 & 1 & 0,5 & 0,5 & 0,4 & 0,4 & 0,4 & 0,4 & 0,4 & 0,4 & 0,4 & 0,6 & 0,8 & 0,5 \\
\hline$E_{4}$ & 0,6 & 0,6 & 0,5 & 1 & 0,7 & 0,7 & 0,7 & 0,6 & 0,6 & 0,6 & 0,6 & 0,6 & 0,5 & 0,5 & 0,7 \\
\hline$E_{5}$ & 0,7 & 0,6 & 0,5 & 0,7 & 1 & 0,6 & 0,7 & 0,6 & 0,6 & 0,8 & 0,6 & 0,8 & 0,5 & 0,5 & 0,6 \\
\hline$E_{6}$ & 0,5 & 0,5 & 0,4 & 0,6 & 0,6 & 1 & 0,7 & 0,7 & 0,6 & 0,8 & 0,6 & 0,8 & 0,5 & 0,5 & 0,6 \\
\hline$E_{7}$ & 0,5 & 0,5 & 0,5 & 0,7 & 0,7 & 0,8 & 1 & 0,8 & 0,7 & 0,8 & 0,6 & 0,7 & 0,6 & 0,5 & 0,7 \\
\hline$E_{8}$ & 0,5 & 0,5 & 0,5 & 0,7 & 0,6 & 0,8 & 0,8 & 1 & 0,7 & 0,7 & 0,6 & 0,7 & 0,7 & 0,5 & 0,7 \\
\hline$E_{9}$ & 0,5 & 0,5 & 0,5 & 0,6 & 0,5 & 0,5 & 0,6 & 0,7 & 1 & 0,5 & 0,6 & 0,7 & 0,7 & 0,6 & 0,6 \\
\hline$E_{10}$ & 0,6 & 0,6 & 0,5 & 0,6 & 0,6 & 0,7 & 0,7 & 0,7 & 0,6 & 1 & 0,7 & 0,7 & 0,5 & 0,5 & 0,7 \\
\hline$E_{11}$ & 0,5 & 0,5 & 0,6 & 0,6 & 0,6 & 0,6 & 0,7 & 0,6 & 0,5 & 0,7 & 1 & 0,7 & 0,5 & 0,5 & 0,8 \\
\hline$E_{12}$ & 0,6 & 0,5 & 0,6 & 0,7 & 0,6 & 0,6 & 0,7 & 0,7 & 0,7 & 0,7 & 0,7 & 1 & 0,6 & 0,6 & 0,8 \\
\hline$E_{13}$ & 0,6 & 0,6 & 0,7 & 0,5 & 0,5 & 0,4 & 0,4 & 0,5 & 0,5 & 0,5 & 0,5 & 0,5 & 1 & 0,8 & 0,5 \\
\hline$E_{14}$ & 0,6 & 0,7 & 0,8 & 0,6 & 0,5 & 0,4 & 0,4 & 0,5 & 0,5 & 0,4 & 0,5 & 0,5 & 0,7 & 1 & 0,4 \\
\hline$E_{15}$ & 0,5 & 0,5 & 0,5 & 0,7 & 0,6 & 0,8 & 0,8 & 0,7 & 0,6 & 0,8 & 0,8 & 0,7 & 0,4 & 0,4 & 1 \\
\hline
\end{tabular}

Fuente: elaboración propia, a partir de las valuaciones vertidas por los expertos. 
Una vez realizada la agregación, se implementó el proceso de composición maxmin con el programa FuzzyLog, esto posibilita agilizar el procesamiento de datos, reduce el margen de error, y permite obtener las incidencias de segundo orden (véase Figura 17).

Figura 17. Matriz de efectos secundarios o de incidencia indirecta

\begin{tabular}{|c|c|c|c|c|c|c|c|c|c|c|c|c|c|c|c|}
\hline & $E_{1}$ & $E_{2}$ & $E_{3}$ & $E_{4}$ & $E_{5}$ & $E_{6}$ & $E_{7}$ & $E_{8}$ & $E_{9}$ & $E_{10}$ & $E_{11}$ & $E_{12}$ & $E_{13}$ & $E_{14}$ & $E_{15}$ \\
\hline$C_{1}$ & 0,6 & 0,7 & 0,7 & 0,6 & 0,6 & 0,6 & 0,6 & 0,6 & 0,6 & 0,7 & 0,6 & 0,6 & 0,6 & 0,7 & 0,6 \\
\hline$C_{2}$ & 0,7 & 0,7 & 0,7 & 0,7 & 0,7 & 0,6 & 0,6 & 0,7 & 0,6 & 0,7 & 0,5 & 0,6 & 0,6 & 0,7 & 0,6 \\
\hline$C_{3}$ & 0,6 & 0,7 & 0,6 & 0,6 & 0,6 & 0,6 & 0,6 & 0,6 & 0,6 & 0,6 & 0,6 & 0,6 & 0,5 & 0,8 & 0,6 \\
\hline$C_{4}$ & 0,6 & 0,7 & 0,7 & 0,7 & 0,6 & 0,6 & 0,6 & 0,6 & 0,6 & 0,7 & 0,6 & 0,6 & 0,7 & 0,7 & 0,6 \\
\hline$C_{5}^{4}$ & 0,6 & 0,7 & 0,7 & 0,6 & 0,6 & 0,6 & 0,6 & 0,6 & 0,7 & 0,6 & 0,6 & 0,6 & 0,8 & 0,8 & 0,6 \\
\hline$C_{6}$ & 0,7 & 0,6 & 0,6 & 0,6 & 0,7 & 0,6 & 0,6 & 0,6 & 0,5 & 0,7 & 0,5 & 0,6 & 0,5 & 0,6 & 0,6 \\
\hline$C_{7}$ & 0,6 & 0,6 & 0,6 & 0,6 & 0,6 & 0,6 & 0,7 & 0,6 & 0,7 & 0,6 & 0,6 & 0,7 & 0,6 & 0,6 & 0,7 \\
\hline$C_{8}$ & 0,6 & 0,6 & 0,6 & 0,7 & 0,6 & 0,7 & 0,7 & 0,8 & 0,6 & 0,6 & 0,7 & 0,7 & 0,6 & 0,6 & 0,8 \\
\hline$C_{9}$ & 0,7 & 0,7 & 0,6 & 0,7 & 0,7 & 0,6 & 0,6 & 1 & 0,5 & 0,7 & 0,5 & 0,6 & 0,5 & 0,5 & 0,6 \\
\hline$C_{10}$ & 0,7 & 0,6 & 0,6 & 0,7 & 0,7 & 0,6 & 0,6 & 0,7 & 0,5 & 0,7 & 0,5 & 0,6 & 0,5 & 0,5 & 0,6 \\
\hline$C_{11}$ & 0,5 & 0,8 & 0,6 & 0,7 & 0,7 & 0,6 & 0,6 & 0,7 & 0,6 & 0,7 & 0,5 & 0,6 & 0,6 & 0,6 & 0,6 \\
\hline$C_{12}$ & 0,5 & 0,6 & 0,6 & 0,6 & 0,6 & 0,6 & 0,6 & 0,6 & 0,5 & 0,6 & 0,5 & 0,6 & 0,5 & 0,5 & 0,5 \\
\hline$C_{13}$ & 0,5 & 0,5 & 0,5 & 0,6 & 0,6 & 0,5 & 0,5 & 0,5 & 0,5 & 0,5 & 0,5 & 0,5 & 0,5 & 0,5 & 0,5 \\
\hline$C_{14}$ & 0,6 & 0,7 & 0,7 & 0,6 & 0,6 & 0,6 & 0,6 & 0,6 & 0,7 & 0,6 & 0,6 & 0,6 & 0,8 & 0,8 & 0,6 \\
\hline
\end{tabular}

El proceso de composición se termina cuando se define la matriz de efectos acumulados $\left[M^{*}\right]$ (véase Figura 18 ).

Figura 18. Incidencias acumuladas causas-efectos

\begin{tabular}{|c|c|c|c|c|c|c|c|c|c|c|c|c|c|c|c|}
\hline & $E_{1}$ & $E_{2}$ & $E_{3}$ & $E_{4}$ & $E_{5}$ & $E_{6}$ & $E_{7}$ & $E_{8}$ & $E_{q}$ & $E_{10}$ & $E_{11}$ & $E_{12}$ & $E_{13}$ & $E_{14}$ & $E_{15}$ \\
\hline$C_{1}$ & 0,6 & 0,7 & 0,7 & 0,6 & 0,6 & 0,7 & 0,7 & 0,7 & 0,6 & 0,7 & 0,7 & 0,7 & 0,7 & 0,7 & 0,7 \\
\hline$C_{2}$ & 0,7 & 0,7 & 0,7 & 0,7 & 0,7 & 0,7 & 0,7 & 0,7 & 0,7 & 0,7 & 0,7 & 0,7 & 0,7 & 0,7 & 0,7 \\
\hline$C_{3}$ & 0,6 & 0,7 & 0,8 & 0,6 & 0,6 & 0,6 & 0,6 & 0,6 & 0,6 & 0,6 & 0,6 & 0,6 & 0,7 & 0,8 & 0,6 \\
\hline$C_{4}$ & 0,6 & 0,7 & 0,7 & 0,7 & 0,7 & 0,7 & 0,7 & 0,7 & 0,6 & 0,7 & 0,7 & 0,7 & 0,7 & 0,7 & 0,7 \\
\hline$C_{5}$ & 0,6 & 0,7 & 0,8 & 0,6 & 0,6 & 0,6 & 0,6 & 0,7 & 0,7 & 0,6 & 0,6 & 0,7 & 0,8 & 0,8 & 0,6 \\
\hline$C_{6}$ & 0,7 & 0,6 & 0,6 & 0,7 & 0,7 & 0,7 & 0,7 & 0,7 & 0,6 & 0,7 & 0,7 & 0,7 & 0,6 & 0,6 & 0,7 \\
\hline$C_{7}$ & 0,6 & 0,6 & 0,6 & 0,7 & 0,7 & 0,7 & 0,7 & 0,7 & 0,7 & 0,7 & 0,7 & 0,7 & 0,7 & 0,6 & 0,7 \\
\hline$C_{8}$ & 0,6 & 0,6 & 0,6 & 0,7 & 0,7 & 0,8 & 0,8 & 0,8 & 0,7 & 0,8 & 0,8 & 0,7 & 0,7 & 0,6 & 0,8 \\
\hline$C_{9}$ & 0,7 & 0,7 & 0,6 & 0,7 & 0,7 & 0,8 & 0,8 & 1 & 0,7 & 0,7 & 0,7 & 0,7 & 0,7 & 0,7 & 0,7 \\
\hline$C_{10}$ & 0,7 & 0,6 & 0,6 & 0,7 & 0,7 & 0,7 & 0,7 & 0,7 & 0,7 & 0,7 & 0,7 & 0,7 & 0,7 & 0,6 & 0,7 \\
\hline$C_{11}$ & 0,7 & 0,8 & 0,6 & 0,7 & 0,7 & 0,7 & 0,7 & 0,7 & 0,7 & 0,7 & 0,7 & 0,7 & 0,7 & 0,7 & 0,7 \\
\hline$C_{12}$ & 0,6 & 0,6 & 0,6 & 0,6 & 0,6 & 0,6 & 0,6 & 0,6 & 0,6 & 0,6 & 0,6 & 0,6 & 0,6 & 0,6 & 0,6 \\
\hline$C_{13}$ & 0,6 & 0,6 & 0,5 & 0,6 & 0,6 & 0,6 & 0,6 & 0,6 & 0,6 & 0,6 & 0,6 & 0,6 & 0,5 & 0,5 & 0,6 \\
\hline$C_{14}$ & 0,6 & 0,7 & 0,8 & 0,6 & 0,6 & 0,6 & 0,6 & 0,7 & 0,7 & 0,6 & 0,6 & 0,7 & 0,8 & 0,8 & 0,6 \\
\hline
\end{tabular}

Fuente: elaboración propia, con el programa FuzzyLog. 


\section{Resultados}

Para evidenciar el grado de olvido de los elementos no considerados en el primer análisis, se calcula la matriz de efectos olvidados $\left[M^{* *}\right]=$ $\left[M^{*}\right]-\left[C E^{\mathrm{A}}\right]$. Esto se logra separando los efectos acumulados manifiestos en la matriz $\mathrm{M}^{*}$, de los directos que aparecen en la matriz original $C E^{\mathrm{A}}$, para ello es suficiente realizar una resta algebraica simple: $\mathrm{M}^{* *}=\mathrm{M}^{*}-C E^{\mathrm{A}}$ (véase Figura 19).

Figura 19. Efectos olvidados en las relaciones de incidencia causa-efecto

\begin{tabular}{|c|c|c|c|c|c|c|c|c|c|c|c|c|c|c|c|}
\hline$\Gamma$ & $E_{1}$ & $E_{2}$ & $E_{3}$ & $E_{4}$ & $E_{5}$ & $E_{6}$ & $E_{7}$ & $E_{8}$ & $E_{9}$ & $E_{10}$ & $E_{11}$ & $E_{12}$ & $E_{13}$ & $E_{14}$ & $E_{15}$ \\
\hline$C_{1}$ & 0,1 & 0 & 0 & 0 & 0 & $\underline{0,3}$ & 0,2 & 0,2 & 0,1 & 0,2 & 0,2 & 0,1 & 0,2 & 0 & 0,1 \\
\hline$C_{2}$ & 0,2 & 0,1 & 0,2 & 0,1 & 0,1 & 0,2 & 0,1 & 0,1 & 0,2 & 0 & 0,2 & 0,2 & $\underline{0,3}$ & 0,2 & 0,2 \\
\hline$C_{3}$ & 0 & 0 & 0,2 & 0,2 & 0,2 & 0,2 & 0,1 & 0,2 & 0,2 & 0,2 & 0 & 0,2 & 0,2 & 0 & 0,1 \\
\hline$C_{4}$ & 0,1 & 0 & 0,1 & 0 & 0,2 & 0,2 & 0,1 & 0,1 & 0 & 0,1 & $\underline{0,3}$ & 0,2 & 0,1 & 0,1 & 0,1 \\
\hline$C_{5}$ & 0 & 0,1 & 0,1 & 0,1 & 0,1 & $\underline{0,3}$ & 0,2 & 0,1 & 0,1 & $\underline{0,3}$ & $\underline{0,3}$ & $\underline{0,3}$ & 0 & 0 & 0,1 \\
\hline$C_{6}$ & 0 & 0,1 & 0,1 & 0,1 & 0 & 0,1 & 0,1 & 0,1 & 0,1 & 0 & 0,2 & 0,1 & 0,2 & 0,2 & 0,2 \\
\hline$C_{7}$ & 0,2 & 0,1 & 0,2 & 0,1 & 0,2 & 0,1 & 0 & 0,1 & 0 & 0,1 & 0,1 & 0 & 0,2 & 0 & 0 \\
\hline$C_{8}$ & 0 & 0,1 & 0,1 & 0 & 0,1 & 0,1 & 0,1 & 0 & 0,1 & 0,2 & 0,1 & 0 & 0,2 & 0 & 0 \\
\hline$C_{9}$ & 0,2 & 0,1 & 0,1 & 0 & 0 & 0,2 & 0,2 & $\underline{0,4}$ & 0,2 & 0 & 0,2 & 0,1 & $\underline{0,3}$ & $\underline{0,3}$ & 0,2 \\
\hline$C_{10}$ & 0,2 & 0,1 & 0,1 & 0,1 & 0,1 & 0,1 & 0,1 & 0,2 & $\underline{0,3}$ & 0 & 0,2 & 0,1 & $\underline{0,3}$ & 0,1 & 0,1 \\
\hline$C_{11}$ & $\underline{0,3}$ & 0 & 0 & 0,2 & $\underline{0,3}$ & $\underline{0,3}$ & 0,2 & 0,2 & $\underline{0,3}$ & 0,1 & $\underline{0,3}$ & $\underline{0,3}$ & $\underline{0,3}$ & 0,2 & $\underline{0,3}$ \\
\hline$C_{12}$ & 0,1 & 0 & 0 & 0,1 & 0,1 & 0,1 & 0,1 & 0,1 & 0,1 & 0,1 & 0,2 & 0,2 & 0,2 & 0,1 & 0,2 \\
\hline$C_{13}$ & 0,1 & 0,1 & 0 & 0 & 0 & 0,1 & 0,1 & 0,1 & 0,1 & 0,1 & 0,2 & 0,1 & 0,1 & 0,1 & 0,1 \\
\hline$C_{14}$ & 0 & 0 & 0,1 & 0,1 & 0,1 & $\underline{0,3}$ & 0,1 & 0,1 & 0 & 0,1 & 0,2 & 0,1 & 0,1 & 0,1 & 0,1 \\
\hline
\end{tabular}

Fuente: elaboración propia, con el programa FuzzyLog.

Es evidente la influencia de $C_{5}, C_{9}$ y $C_{11}$, por la cantidad de efectos distribuidos entre un número considerable de efectos. Esta condición presupone la necesidad de identificar las variables interpuestas, por medio de las cuales la relación de incidencia se hace presente (véase Figura 20). 
Figura 20. Variables con grado de olvido ${ }^{6}$

\begin{tabular}{|c|c|c|c|c|}
\hline Causa & Efecto & Valor inicial & Valor acumulado & Valor olvidado \\
\hline $\begin{array}{l}\text { Instituciones } \\
\text { económicas }\end{array}$ & $\begin{array}{l}\text { Incremento } \\
\text { delincuencia }\end{array}$ & $\mu i\left(c_{1} \rightarrow e_{6}\right)=0,4$ & $\mu a\left(c_{1} \rightarrow e_{6}\right)=0,7$ & $\mu o\left(c_{1} \rightarrow e_{6}\right)=0,3$ \\
\hline $\begin{array}{l}\text { Instituciones } \\
\text { políticas }\end{array}$ & $\begin{array}{l}\text { Progreso } \\
\text { técnico }\end{array}$ & $\mu i\left(c_{2} \rightarrow e_{13}\right)=0,4$ & $\mu a\left(c_{2} \rightarrow e_{13}\right)=0,7$ & $\mu o\left(c_{2} \rightarrow e_{13}\right)=0,3$ \\
\hline $\begin{array}{l}\text { Presupuesto } \\
\text { público }\end{array}$ & $\begin{array}{c}\text { Problemas } \\
\text { laborales }\end{array}$ & $\mu i\left(c_{4} \rightarrow e_{11}\right)=0$, & $\mu a\left(c_{4} \rightarrow e_{11}\right)=0,8$ & $\mu o\left(c_{4} \rightarrow e_{11}\right)=0,3$ \\
\hline Tecnología & $\begin{array}{l}\text { Incremento } \\
\text { delincuencia }\end{array}$ & $\mu i\left(c_{5} \rightarrow e_{6}\right)=0,3$ & $\mu a\left(c_{5} \rightarrow e_{6}\right)=0,8$ & $\mu o\left(c_{5} \rightarrow e_{6}\right)=0,3$ \\
\hline Tecnología & $\begin{array}{l}\text { Inestabilidad } \\
\text { social }\end{array}$ & $\mu i\left(c_{5} \rightarrow e_{10}\right)=0,2$ & $\mu a\left(c_{5} \rightarrow e_{10}\right)=0,7$ & $\mu o\left(c_{5} \rightarrow e_{10}\right)=0,3$ \\
\hline Tecnología & $\begin{array}{c}\text { Problemas } \\
\text { laborales }\end{array}$ & $\mu i\left(c_{5} \rightarrow e_{11}\right)=0,2$ & $\mu a\left(c_{5} \rightarrow e_{11}\right)=0,7$ & $\mu o\left(c_{5} \rightarrow e_{11}\right)=0,3$ \\
\hline Tecnología & $\begin{array}{l}\text { Disminución } \\
\text { inversión }\end{array}$ & $\mu i\left(c_{5} \rightarrow e_{12}\right)=0,2$ & $\mu a\left(c_{5} \rightarrow e_{12}\right)=0,7$ & $\mu o\left(c_{5} \rightarrow e_{12}\right.$ \\
\hline $\begin{array}{c}\text { Frágil } \\
\text { democracia }\end{array}$ & $\begin{array}{c}\text { Retraso } \\
\text { educativo }\end{array}$ & $\mu i\left(c_{9} \rightarrow e_{8}\right)=0,2$ & $\mu a\left(c_{9} \rightarrow e_{8}\right)=0,7$ & $\mu o\left(c_{9} \rightarrow e_{8}\right)=0,4$ \\
\hline $\begin{array}{c}\text { Frágil } \\
\text { democracia }\end{array}$ & $\begin{array}{l}\text { Progreso } \\
\text { técnico }\end{array}$ & $\mu i\left(c_{9} \rightarrow e_{13}\right)=0,2$ & $\mu a\left(c_{9} \rightarrow e_{13}\right)=0,7$ & $\mu o\left(c_{9} \rightarrow e_{13}\right)=0,3$ \\
\hline $\begin{array}{c}\text { Frágil } \\
\text { democracia }\end{array}$ & $\begin{array}{l}\text { Incremento } \\
\text { productividad }\end{array}$ & $\mu i\left(c_{9} \rightarrow e_{14}\right)=0,2$ & $\mu a\left(c_{9} \rightarrow e_{14}\right)=0,7$ & $\mu o\left(c_{9} \rightarrow e_{14}\right.$ \\
\hline $\begin{array}{l}\text { Conflictos } \\
\text { grupos de } \\
\text { poder }\end{array}$ & $\begin{array}{l}\text { Estancamiento } \\
\text { tecnológico }\end{array}$ & $\mu i\left(c_{10} \rightarrow e_{9}\right)=0,2$ & $\mu a\left(c_{10} \rightarrow e_{9}\right)=0,7$ & $\mu o\left(c_{10} \rightarrow e_{9}\right)=0,3$ \\
\hline $\begin{array}{l}\text { Conflictos } \\
\text { grupos de } \\
\text { poder }\end{array}$ & $\begin{array}{l}\text { Progreso } \\
\text { técnico }\end{array}$ & $\mu i\left(c_{10} \rightarrow e_{13}\right)=0,2$ & $\mu a\left(c_{10} \rightarrow e_{13}\right.$ & $\mu o\left(c_{10} \rightarrow e_{13}\right)=0,3$ \\
\hline $\begin{array}{l}\text { Participación } \\
\text { ciudadana }\end{array}$ & $\begin{array}{c}\text { Riqueza } \\
\text { individual }\end{array}$ & $\mu i\left(c_{11} \rightarrow e_{1}\right)=0,2$ & $\mu a\left(c_{11} \rightarrow e_{1}\right)=0,7$ & $\mu o\left(c_{11} \rightarrow e_{1}\right)=0,3$ \\
\hline $\begin{array}{l}\text { Participación } \\
\text { ciudadana }\end{array}$ & $\begin{array}{l}\text { Concentración } \\
\text { de riqueza }\end{array}$ & $\mu i\left(c_{11} \rightarrow e_{5}\right)=0,2$ & $\mu a\left(c_{11} \rightarrow e_{5}\right)=0,7$ & $\mu o\left(c_{11} \rightarrow e_{5}\right)=0,3$ \\
\hline $\begin{array}{l}\text { Participación } \\
\text { ciudadana }\end{array}$ & $\begin{array}{l}\text { Incremento } \\
\text { delincuencia }\end{array}$ & $\mu i\left(c_{11} \rightarrow e_{6}\right)=0,2$ & $\mu a\left(c_{11} \rightarrow e_{6}\right)=0,7$ & $\mu o\left(c_{11} \rightarrow e_{6}\right)=0,3$ \\
\hline $\begin{array}{l}\text { Participación } \\
\text { ciudadana }\end{array}$ & $\begin{array}{l}\text { Estancamiento } \\
\text { tecnológico }\end{array}$ & $\mu i\left(c_{11} \rightarrow e_{9}\right)=0,2$ & $\mu a\left(c_{11} \rightarrow e_{9}\right)=0,7$ & $\mu o\left(c_{11} \rightarrow e_{9}\right)=0,3$ \\
\hline $\begin{array}{l}\text { Participación } \\
\text { ciudadana }\end{array}$ & $\begin{array}{l}\text { Problemas } \\
\text { laborales }\end{array}$ & $\mu i\left(c_{11} \rightarrow e_{11}\right)=0,2$ & $\mu a\left(c_{11} \rightarrow e_{11}\right)=0,7$ & $\mu o\left(c_{11} \rightarrow e_{11}\right)=0,3$ \\
\hline $\begin{array}{l}\text { Participación } \\
\text { ciudadana }\end{array}$ & $\begin{array}{l}\text { Disminución } \\
\text { de inversión }\end{array}$ & $\mu i\left(c_{11} \rightarrow e_{12}\right)=0,2$ & $\mu a\left(c_{11} \rightarrow e_{12}\right)=0,7$ & $\mu o\left(c_{11} \rightarrow e_{12}\right)=0,3$ \\
\hline $\begin{array}{l}\text { Participación } \\
\text { ciudadana }\end{array}$ & $\begin{array}{l}\text { Progreso } \\
\text { técnico }\end{array}$ & $\mu i\left(c_{11} \rightarrow e_{13}\right)=0,2$ & $\mu a\left(c_{11} \rightarrow e_{13}\right)=0,7$ & $\mu o\left(c_{11} \rightarrow e_{13}\right)=0,3$ \\
\hline $\begin{array}{l}\text { Participación } \\
\text { ciudadana }\end{array}$ & Desempleo & $\mu i\left(c_{11} \rightarrow e_{15}\right)=0,2$ & $\mu a\left(c_{11} \rightarrow e_{15}\right)=0,7$ & $\mu o\left(c_{11} \rightarrow e_{15}\right)=0,3$ \\
\hline $\begin{array}{l}\text { Inversión en } \\
I \& D\end{array}$ & $\begin{array}{l}\text { Incremento } \\
\text { delincuencia }\end{array}$ & $\mu i\left(c_{14} \rightarrow e_{6}\right)=0,2$ & $\mu a\left(c_{14} \rightarrow e_{6}\right)=0,7$ & $\mu o\left(c_{14} \rightarrow e_{6}\right)=0,3$ \\
\hline
\end{tabular}

Fuente: elaboración propia, a partir de los resultados obtenidos con el programa FuzzyLog.

6 Las ecuaciones muestran las relaciones de incidencia concreta y sistemática. Los valores se obtienen de los resultados arrojados por la matriz de efectos olvidados, cuya lectura se realiza de la siguiente manera:

$\mu i\left(c_{1} \rightarrow e_{6}\right)=0,4$ el valor inicial de la causa 1 sobre el efecto 6 , que de acuerdo con la apreciación de los expertos es de 0,4.

$\mu a\left(c_{1} \rightarrow e_{6}\right)=0,7$ el valor acumulado de la causa 1 sobre el efecto 6 es de 0,7 ; se obtiene al aplicar el proceso de composición maxmin.

$\mu o\left(c_{1} \rightarrow e_{6}\right)=0,3$ por tanto, el valor olvidado de la causa 1 sobre el efecto 6 es de 0,3 . 
En virtud de que los resultados muestran una cantidad relevante de variables con grado de olvido pequeño, es conveniente enfatizar el análisis en los más representativos.

\section{Determinación de gráficos de incidencia, relaciones interpuestas y grados de olvido}

Representar las relaciones entre el conjunto de causas y efectos de manera gráfica permite comprender con mayor claridad los olvidos de los integrantes de la elite del poder que dirigen las instituciones, para de esa forma sugerir la ruta de incidencia, que facilite el diseño de políticas públicas orientadas a mejorar el desempeño de la economía sinaloense en el mediano y largo plazo (véase Figura 21).

Figura 21. Variables con grado de olvido, relación interpuesta

\begin{tabular}{|c|c|c|}
\hline $\begin{array}{l}\text { Variable con grado } \\
\text { de olvido }\end{array}$ & $\begin{array}{l}\text { Variable } \\
\text { interpuesta }\end{array}$ & $\begin{array}{l}\text { Variable } \\
\text { afectada }\end{array}$ \\
\hline $\begin{array}{l}\text { Instituciones } \\
\text { económicas }\end{array}$ & Instituciones políticas & Incremento de la delincuencia \\
\hline Instituciones políticas & Instituciones económicas & Progreso técnico \\
\hline Presupuesto público & Instituciones políticas & Problemas laborales \\
\hline Tecnología & $\begin{array}{c}\text { Instituciones económicas } \\
\text { Tecnología } \\
\text { Baja inversión en capital } \\
\text { humano }\end{array}$ & $\begin{array}{l}\text { Incremento de la delincuencia } \\
\text { Problemas laborales } \\
\text { Disminución de la inversión }\end{array}$ \\
\hline Frágil democracia & $\begin{array}{l}\text { Frágil democracia } \\
\text { Prosperidad social }\end{array}$ & $\begin{array}{c}\text { Retraso educativo } \\
\text { Incremento de productividad }\end{array}$ \\
\hline $\begin{array}{l}\text { Conflictos de grupos } \\
\text { de poder }\end{array}$ & Grupos de poder & Estancamiento tecnológico \\
\hline $\begin{array}{c}\text { Participación } \\
\text { ciudadana }\end{array}$ & $\begin{array}{l}\text { Frágil democracia } \\
\text { Frágil democracia } \\
\text { Instituciones políticas } \\
\text { Instituciones políticas } \\
\text { Frágil democracia } \\
\text { Desigualdad regional }\end{array}$ & $\begin{array}{c}\text { Riqueza individual } \\
\text { Concentración de riqueza } \\
\text { Incremento de delincuencia } \\
\text { Problemas laborales } \\
\text { Disminución de inversión } \\
\text { Desempleo }\end{array}$ \\
\hline Inversión en I \&D & Instituciones económicas & Incremento de delincuencia \\
\hline
\end{tabular}

Fuente: elaboración propia. 
Los expertos de la elite del poder en Sinaloa afirman que las instituciones económicas tienen una incidencia de 0,4 sobre el incremento de la delincuencia, no obstante, se aprecia que éstas tienen una de 0,7 sobre las instituciones políticas, que a su vez influyen en la inestabilidad social, del mismo grado. Por tanto, de manera indirecta se detecta la existencia de un grado de olvido de 0,3 con respecto al valor asignado inicialmente. Considerar los elementos interpuestos, entre causas y efectos, les facilita a los integrantes del poder político de jure, diseñar las políticas públicas promotoras del crecimiento económico (véase Figura 22 y 23 ).

Figura 22. Instituciones económicas-incremento de la delincuencia

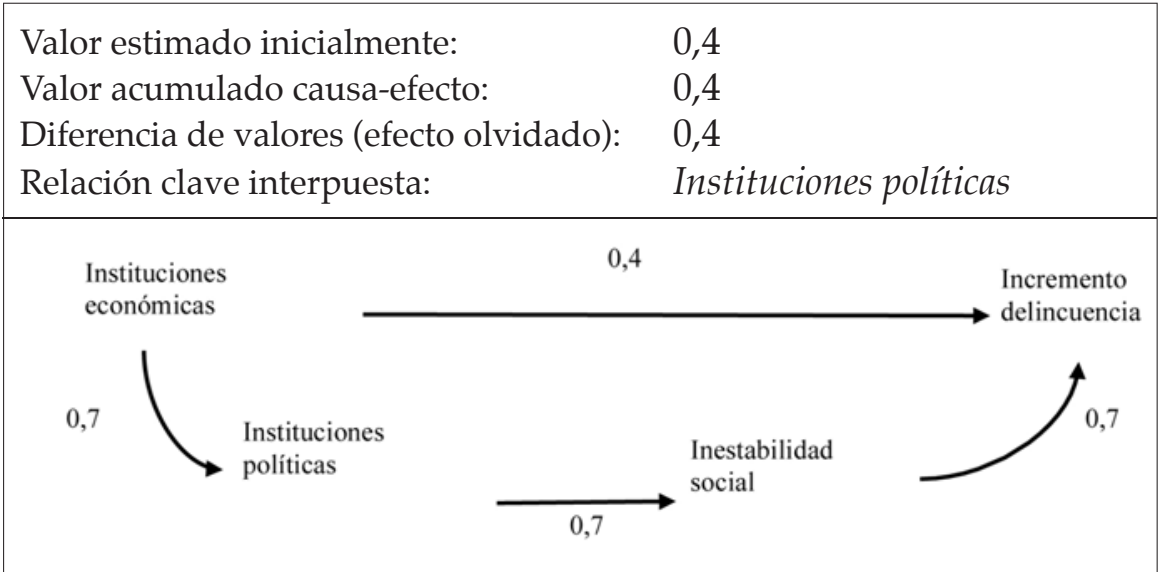

Fuente: elaboración propia.

López et al. (1998) afirman que las políticas públicas que promueven la inversión en tecnología tienen una influencia significativa en el crecimiento al incidir en las instituciones económicas, el desarrollo del capital humano y la investigación y desarrollo. Promover el progreso tecnológico depende cada vez más de la mejora de los cauces institucionales, que posibiliten una intervención pública efectiva en dicha gestión; es decir, una que pueda reflejar mejor la voluntad de los ciudadanos. 


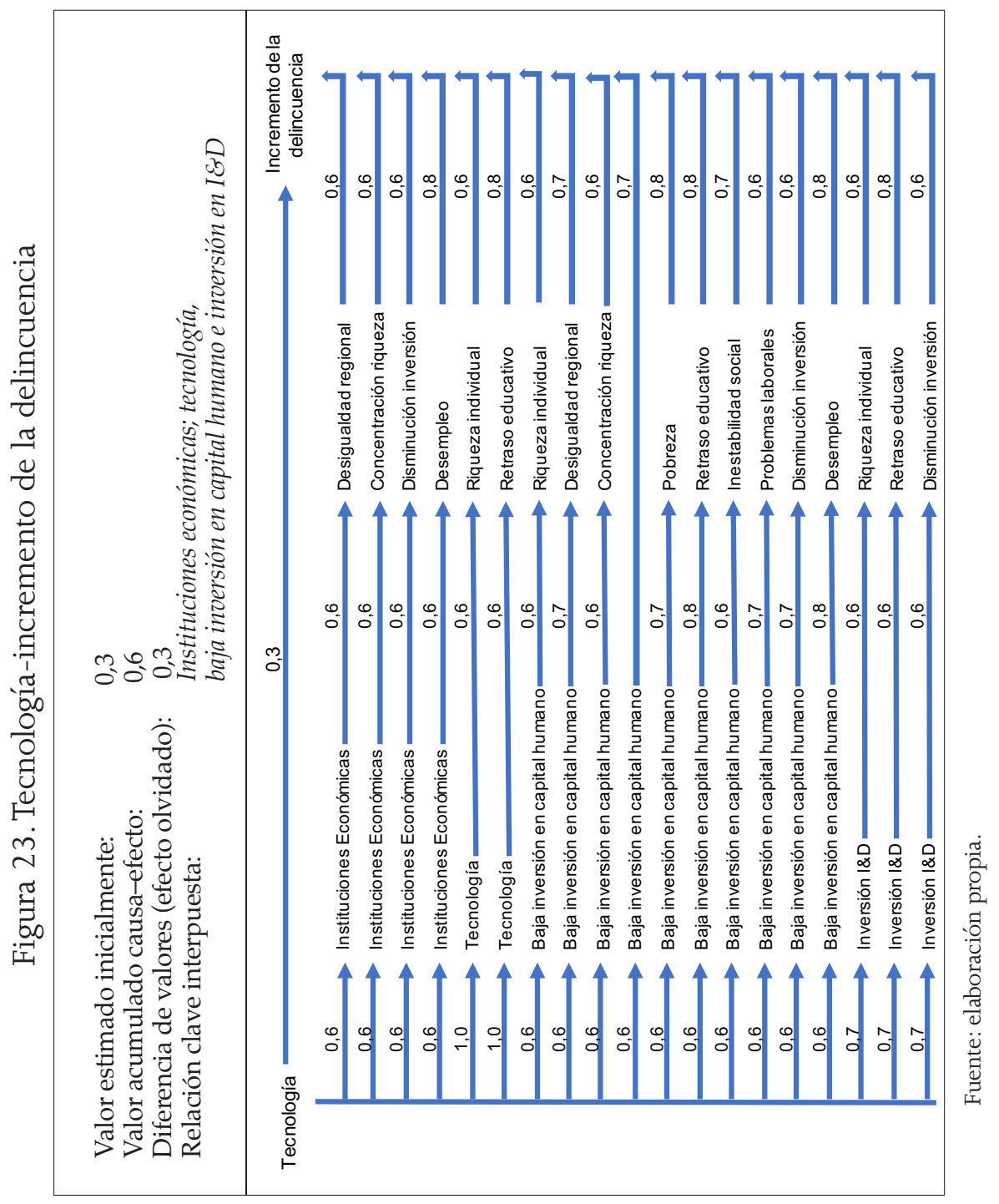


Figura 24. Frágil democracia-retraso educativo

Valor estimado inicialmente:

0,7

Valor acumulado causa-efecto:

1,0

Diferencia de valores (efecto olvidado):

0,4

Relación clave interpuesta:

Frágil democracia

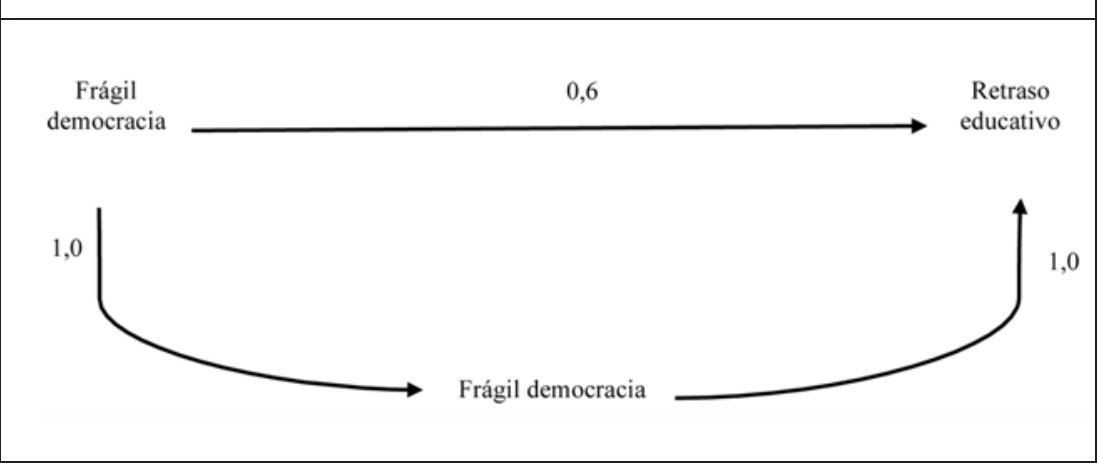

Fuente: elaboración propia.

De la Figura 24 se deduce que de la ausencia de una democracia fuerte se derivan fenómenos interrelacionados como la concentración del ingreso, los grupos de poder y la falta de trasparencia, cuya presencia, a su vez, potencializan la pobreza de oportunidades y el retraso educativo. Ello comprueba el postulado de Friedman (1962), quien apunta que la calidad de la democracia incide en el crecimiento; argumenta que la fortaleza democrática se manifiesta en la energía de las instituciones económicas. La incrustación institucional de los grupos de poder perjudica la libre competencia. Las elites son capaces de acordar y crear una coalición para apropiarse de la trayectoria y de los beneficios del crecimiento, y también propiciar una asignación inequitativa de los recursos.

En la Figura 25 se aprecia una valuación de 0,6, sin embargo, en el proceso de composición, donde está como elemento interpuesto la variable frágil democracia, con una incidencia de 1, por tanto, tiene un grado de olvido de 0,4 . 


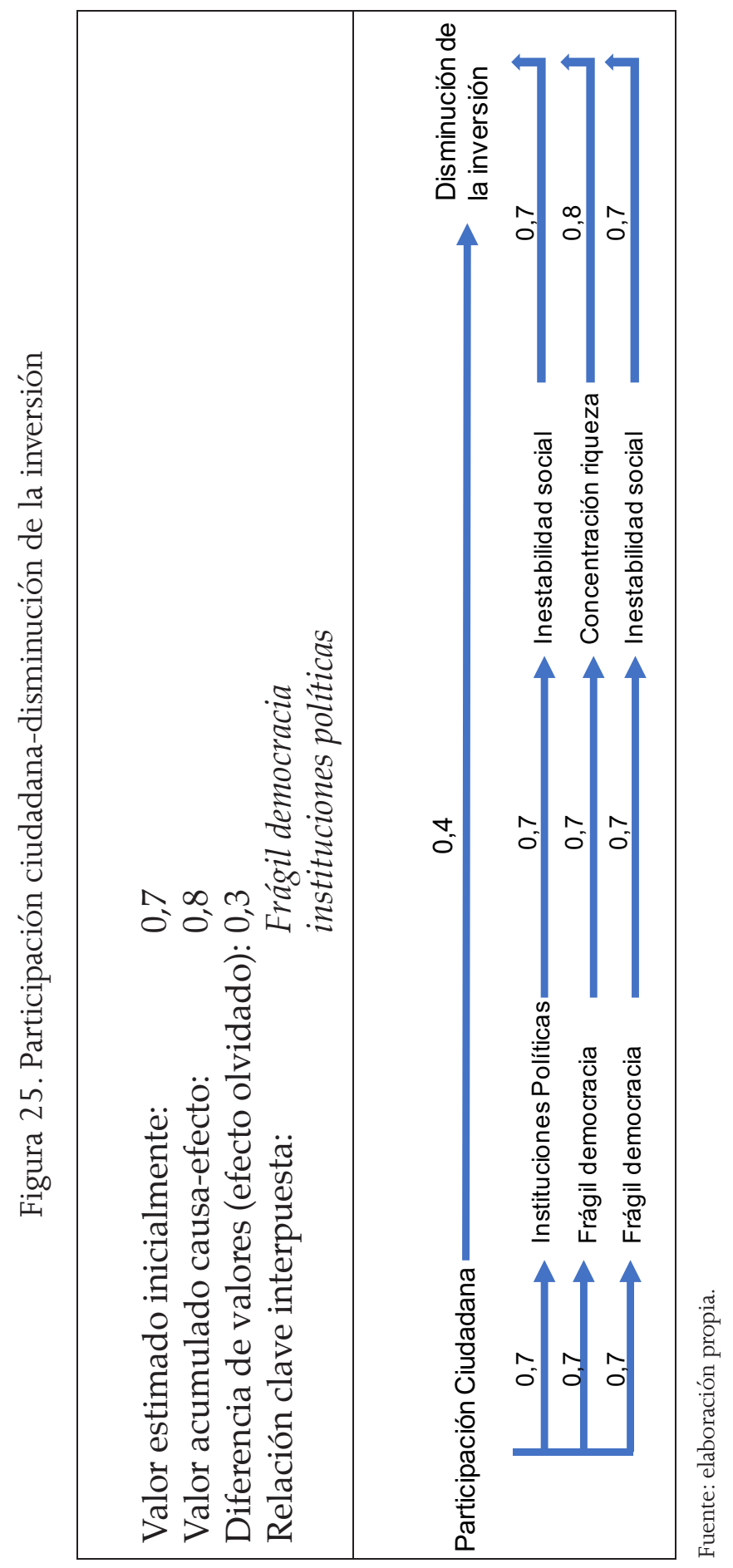




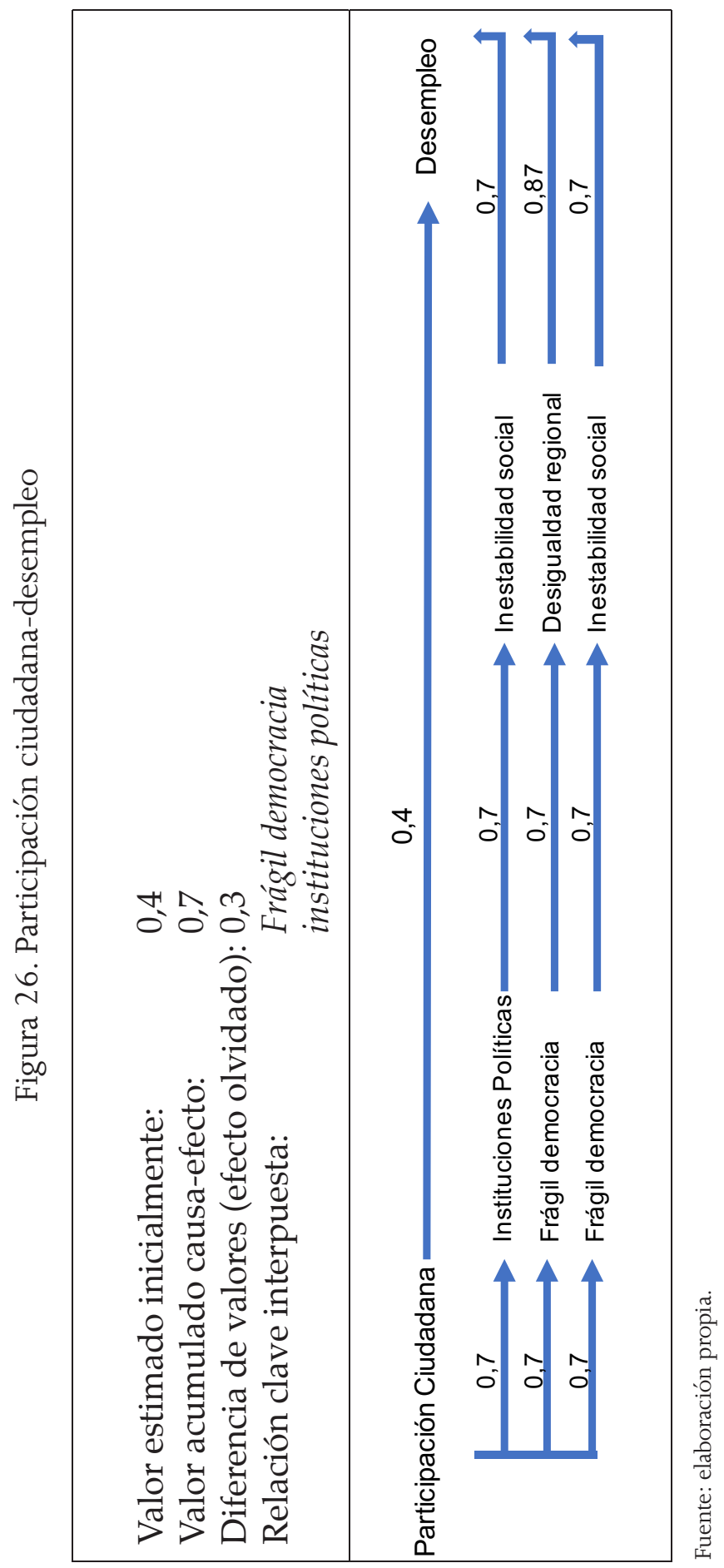


Los resultados obtenidos en la matriz de olvidos son congruentes con los postulados teóricos. De lo observado en las Figuras 24, 25 y 26 se deduce que la ausencia de una democracia fuerte incide en otros fenómenos como la concentración de la riqueza, la inestabilidad social y la desigualdad regional, cuya acumulación de valores determina el grado de olvido de las variables: retraso educativo, disminución de la inversión y desempleo. Los valores asignados contrastan con las percepciones originales de los tomadores de decisiones, con los efectos de segunda generación no contemplados. Esos valores representan la gradualidad del olvido en el diseño de acciones tendientes a promover el crecimiento.

\section{Reflexiones finales}

Solow (1956) considera que el crecimiento no se debe atribuir preponderantemente a la acumulación de capital, sino al avance tecnológico, vía el aprendizaje de mejorar el "cómo hacer las cosas”. Si bien el aumento de la productividad evidencia la repercusión de los grandes descubrimientos, en mayor medida es resultado de cambios pequeños y graduales. De esa forma, Stiglitz y Greenwald (2015) afirman que tiene sentido centrar la atención en cómo "aprenden" las sociedades, y qué hacen para promover el aprendizaje, que incluye el impulso de cómo "aprender a aprender".

Los resultados de la investigación concuerdan con los postulados de Solow (1956), North (1990), Barro (1999), Sala-i-Martin (2000), Acemoglu et al. (2005), Stiglitz y Greenwald (2015) y Ros (2008). Se observa que las variables tecnología, frágil democracia, participación ciudadana e inversión en investigación y desarrollo obstruyen el crecimiento al acumular grados de olvido, lo cual denota un esfuerzo institucional insuficiente. Asimismo, se demuestra que estas variables tienen una relación de incidencia sobre la inversión limitada en capital humano, investigación y desarrollo y tecnología, variables determinadas como elementos interpuestos, cuya incidencia es mayor sobre los efectos: bajo progreso técnico, retraso educativo, estancamiento tecnológico y delincuencia creciente, que ocultan los olvidos iniciales, que explican la debilidad del crecimiento de mediano y largo plazo y, en consecuencia, el aumento en la pobreza y la inequidad del ingreso. 
Stiglitz y Greenwald (2015) argumentan que la evaluación de la política pública orientada a incentivar la dinámica productiva, a través de las lentes del aprendizaje ofrece una perspectiva diferente, y así lo evidencian los resultados. Los avances en tecnología, así como en "aprender a hacer las cosas mejor" son determinantes significativos en el aumento de la productividad. La metodología de los efectos olvidados permite respaldar la validez de tal afirmación, pues según los resultados la inversión en tecnología y en investigación y desarrollo están entre las variables que explican con mayor incidencia la debilidad del desempeño de la economía sinaloense.

\section{Bibliografía}

Acemoglu, Daron. 2009. Introduction to modern economic growth. Reino Unido: Princeton University Press.

Acemoglu, Daron, Simon Johnson y James Robinson. 2005. Institutions as a fundamental cause of long-run growth. En Handbook of economic growth, volumen 1A, editado por Philippe Aghion y S. N. Durlauf, 385-472. Ámsterdam: Elsevier North-Holland.

Barcellos, L. 2010. Modelos de gestión aplicados a la sostenibilidad empresarial. Barcelona: Universitat de Barcelona.

Barro, Robert J. 1999. Determinants of economic growth. Cambridge: MIT Press.

Burdeau, Georges. 1966. Traité de science politique. París: Pichon-Durant. Cass, David. 1965. Optimum growth in an aggregative model of capital accumulation. Review of Economic Studies 32 (3): 233-240.

CEFP de la Cámara de Diputados del H. Congreso de la Unión. 2016. http://www.cefp.gob.mx/

CONEVAL. 2014. Anexo estadístico. http://www.coneval.gob.mx/ Medicion/MP/Paginas/AE_pobreza_2014.aspx (15 de octubre de 2015). 
Duverger, Maurice. 1997. Ciencia política. México: Hemisferio.

Easton, David. 1953. The political system. Nueva York: Knopf.

Ericsson, K., M. Prietula y E. Cokely. 2007. La formación de un experto. Harvard Business Review. América Latina 8: 113-120.

Espinoza, Víctor A. y Alejandro Monsiváis. 2012. El deterioro de la democracia: consideraciones sobre el régimen político, lo público y la ciudadanía en México. Mexico: El Colegio de la Frontera Norte.

Foucault, Michel. 1988. El sujeto y el poder. Revista Mexicana de Sociología 50 (3): 3-20.

Friedman, Milton. 1962. Capitalism and freedom. Chicago: Chicago University Press.

Gento, Ángel, Luisa L. Lazzari y Emilio A. Machado. 1999. Reflexiones acerca de las matrices de incidencia y la recuperación de los efectos olvidados. Cuadernos del CIMBAGE (4): 11-27.

Gil Aluja, Jaime. 2005. La matemática borrosa en la economía y gestión de empresas. Matematicalia: Revista Digital de Divulgación Matemática de la Real Sociedad Matemática Española 1 (3): 5.

Gil Aluja, Jaime. 1999. Elementos para una teoría de la decisión en la incertidumbre. Londres: Vigo Villadoiro.

Gil Aluja, Jaime. 1996. Towards a new paradigm of invesment selection in uncertainty. Fuzzy Sets and Systems 84 (2): 187-197.

Gil Aluja, Jaime et al. 2004. Aproximación metodológica a la optimización en la incertidumbre. Monográfico (2): 23-49.

Gil Lafuente, Ana María. 1993. El análisis financiero en la incertidumbre. Barcelona: Ariel. 
Gil Lafuente, Ana María y Luis Bassa. 2011. Identificación de los atributos contemplados por los clientes en una estrategia CRM utilizando el modelo de efectos olvidados. Cuadernos del CIMBAGE (13): 107-127.

Gil Lafuente, Ana María y Jaime Gil Lafuente. 2007. Modelos y algoritmos para tratar la creatividad en la gestión empresarial. España: European Academic Publishers-Milladoiro.

Hernández, Rogelio. 1998. Amistades, compromisos y lealtades: líderes y grupos políticos en el Estado de México 1942-1993. México: El Colegio de México.

Hume, David. 1980. Acerca de las facciones:teoría y sociología crítica de los partidos políticos. Barcelona: Anagrama.

Ibarra, S. Jorge. 2013. Entorno político y dependencia financiera de los estados mexicanos. Gestión y Política Pública (2): 3-44.

Inda, Graciela y Celia Duek. 2005. Escritos de sociología ETHOS. Aposta Revista de Ciencias Sociales 23 (3).

INEGI. 2016. Banco de información INEGI. http://www3.inegi.org. $\mathrm{mx} /$ sistemas/biinegi/ (20 de enero de 2016).

INEGI. 2014. Módulo de condiciones socioeconómicas de le Encuesta nacional de ingresos y gastos de los hogares (ENIGH). http:// www.inegi.org.mx/est/contenidos/proyectos/encuestas/hogares/modulos/mcs/mcs2014/default.aspx (16 de noviembre de 2015).

Jusidman, Clara. 2009. Desigualdad y política social en México. Nueva Sociedad (220): 190-206.

Kauffmann, Arnold y Jaime Gil Aluja. 1993. Técnicas especiales para la gestión de expertos. Santiago de Compostela: Milladoiro. 
Kauffmann, Arnold y Jaime Gil Aluja. 1990. Las matemáticas del azar y de la incertidumbre; elementos básicos para su aplicación en economía. Madrid: Centro de Estudios Ramón Areces.

Kauffmann, Arnold y Jaime Gil Aluja. 1988. Modelos para la investigación de efectos olvidados. Vigo: Milladoiro.

Kauffmann, Arnold y Jaime Gil Aluja. 1987. Técnicas operativas de gestión para el tratamiento de la incertidumbre. Barcelona: Hispano Europea, S. A.

Knoke, David. 1994. Networks of elite structure and decision making. Sage Focus Editions (171): 274-274.

Koopmas, Tjalling C. 1965. Economic growth at a maximal rate. Quarterly Journal of Economics 78 (3): 355-394.

Krugman, Paul. 1999. Development, geography, and economic theory. Cambridge: MIT Press.

Lomnitz, Larissa y Gil Mendieta. 2002. El neoliberalismo y los cambios en la elite de poder en México. Redes: Revista Hispana para el Análisis de Redes Sociales 1 (5).

López, C., J. Méndez y O. Todt. 1998. Participación pública en política tecnológica: problemas y perspectivas. Arbor 159 (627): 279.

Lucas, Robert. 1988. On the mechanics of economic development. Journal of Monetary Economics 22 (1): 3-42.

Maritain, Jacques. 1983. El hombre y el Estado. Madrid: Ediciones Encuentro (12).

Moore, George. 1994. The structure of a national elite network. American Sociological Review (44): 673-692.

North, Douglass C. 1994. Institutional change: a framework for analysis. En Institutional change: theory and empirical findings, editado por SvenErik Sjöstrand, 35-46. Nueva York: Armonk. 
North, Douglass C. 1993. Prize lecture: economic performance through time. Nobelprize.org. Nobel Media AB 2014. http://www.nobelprize.org/nobel_prizes/economic-sciences/laureates/1993/ north-lecture.html (20 de julio de 2015).

North, Douglass C. 1990. Institutions, institutional change, and economic perfomance. Nueva York: Cambridge University Press.

North, Douglass C. 1981. Structure and change in economic history. Nueva York: Norton \& Co.

North, Douglass C. y Robert Thomas. 1973. The rise of the western world: a new economic history. Cambridge: Cambridge University Press.

Plumpler, Thomas y Chistian. W Martin. 2003. Democracy, government spending, and economic growth: a political-economic explanation of the Barro-effect. Public Choice 117 (1-2): 27-50.

Romer, Paul M. 1986. Increasing returns and long run growth. Journal of Political Economy 94 (5): 1002-1037.

Ros, Jaime. 2008. La desaceleración del crecimiento económico en México desde 1982. El Trimestre Económico 75 (299): 537-560.

Sala-i-Martin, Xavier. 2000. Apuntes de crecimiento económico. España: Antoni Bosch.

Salazar, R. 2012. The Mexican peso: exchange risk coverage management through the forgotten effects theory. Journal of Economics, Finance and Administrative Science 17: 53-74.

Schmidt, Samuel y Jorge Gil Mendieta. 2002. Los grupos de poder en México: recomposiciones y alianzas. Estudios Interdisciplinarios de América Latina y el Caribe 14 (2).

Solow, Robert. 1956. A contribution to the theory of economic growth. Quarterly Journal of Economics 70 (1): 65-94. 
Stiglitz, Joseph E. y Bruce C. Greenwald. 2015. La creación de una sociedad del aprendizaje. México: Paidós.

Swan, W. Trevor. 1956. Economic growth and capital accumulation. Economic Record 32 (2): 334-361.

Swyngedouw, Erik. 2000. Elite power, global forces, and the political economy of 'glocal' development. En The Oxford handbook of economic geography, editado por Gordon L. Clark, Maryann P. Feldaman y Meric S. Gertler, 541-558. Nueva York: Oxford University Press.

Tichy, G. 2004. The over-optimism among experts in assessment and foresight. Technological Forecasting and Social Change 71 (4): 341-363.

Villarreal, Héctor. 2013. Roderic Ai Camp: el reclutamiento político en México. METAPOLÍTICA 17 ( 83): 50-54.

Zadeh, Lofty A. 1965. Fuzzy Set. Information and Control 8 (3): 338-353. 